\title{
Optimal consumption and portfolio with both fixed and proportional transaction costs
}

\author{
Bernt Øksendal ${ }^{1,2} \quad$ Agnès Sulem ${ }^{3}$
}

May 2, 2001

\begin{abstract}
We consider a market model with one riskfree and one risky asset, in which the dynamics of the risky asset is governed by a geometric Brownian motion. In this market we consider an investor who consumes from the bank account and who has the opportunity at any time to transfer funds between the two assets. We suppose that these transfers involve a fixed transaction cost $k>0$, independent of the size of the transaction, plus a cost proportional to the size of the transaction.

The objective is to maximize the cumulative expected utility of consumption over a planning horizon. We formulate this problem as a combined stochastic control/impulse control problem, which in turn leads to a (nonlinear) quasivariational Hamilton-Jacobi-Bellman inequality (QVHJBI). We prove that the value function is the unique viscosity solution of this QVHJBI. Finally numerical results are presented.
\end{abstract}

\section{Introduction}

Let $(\Omega, \mathcal{F}, P)$ be a probability space with a given filtration $\left\{\mathcal{F}_{t}\right\}_{t \geq 0}$. We denote by $X(t)$ the amount of money the investor has in the bank at time $t$ and by $Y(t)$ the amount of money invested in the risky asset at time $t$. We assume that in the absence of consumption and transactions the process $X(t)$ grows deterministically at exponential rate $r$, while $Y(t)$ is a geometric Brownian motion, i.e.

$$
d X(t)=r X(t) d t ; \quad X(0)=x
$$

\footnotetext{
${ }^{1}$ Dept. of Mathematics, University of Oslo, P. O. Box 1053 Blindern, N-0316 Oslo, Norway email: oksendal@math.uio.no

${ }^{2}$ Norwegian School of Economics and Business Administration, Helleveien 30, N-5045 Bergen, Norway

${ }^{3}$ INRIA, Domaine de Voluceau-Rocquencourt B.P. 105, F-78153 Le Chesnay Cedex, France email: Agnes.Sulem@inria.fr
} 


$$
d Y(t)=\alpha Y(t) d t+\sigma Y(t) d W(t) ; \quad Y(0)=y
$$

where $W(t)$ is a 1-dimensional $\mathcal{F}_{t}$-Brownian motion and $\alpha>r>0$ and $\sigma \neq 0$ are constants.

Suppose that at any time $t$ the investor is free to choose a consumption rate $c(t) \geq 0$. This consumption is automatically drawn from the bank account holding with no extra costs. Moreover, at any time the investor can decide to transfer money from the bank account to the stock and conversely. Assume that a purchase of size $\ell$ of stocks incurs a transaction cost consisting of a sum of a fixed cost $k>0$ (independent of the size of the transaction) plus a cost $\lambda \ell$ proportional to the transaction $(\lambda \geq 0)$. These costs are drawn from the bank account. Similarly a sale of size $m$ of stocks incures the fixed cost $K>0$ plus the proportional cost $\mu m(\mu \geq 0)$. For simplicity we will assume that $K=k$ and $\mu=\lambda$. In this context the investor will only change his portfolio finitely many times in any finite time interval. The control of the investor will consist of a combination of a regular stochastic control $c(t)$ and an impulse control $v=\left(\tau_{1}, \tau_{2}, \ldots ; \xi_{1}, \xi_{2}, \ldots\right)$. Here $0 \leq \tau_{1}<\tau_{2}<\ldots$ are $\mathcal{F}_{t}$-stopping times giving the times when the investor decides to change his portfolio and $\left\{\xi_{j} \in \mathbf{R} ; j=1,2, \ldots\right\}$ are $\mathcal{F}_{\tau_{j}}$-measurable random variables giving the sizes of the transactions at these times. We assume that

$$
c(t) \text { is } \mathcal{F}_{t^{-}} \text {adapted, } c(t, \omega) \geq 0 \text { and } \lim _{j \rightarrow \infty} \tau_{j}=\infty \text { a.s. }
$$

(possibly $\tau_{n}=\infty$ a.s. for some $n<\infty$ ).

If such a control $w=(c, v)$ is applied to the system $(X(t), Y(t))$ it gets the form

$$
\begin{aligned}
& d X(t)=(r X(t)-c(t)) d t ; \quad \tau_{i} \leq t<\tau_{i+1} \\
& d Y(t)=\alpha Y(t) d t+\sigma Y(t) d W(t) ; \quad \tau_{i} \leq t<\tau_{i+1} \\
& X\left(\tau_{i+1}\right)=X\left(\tau_{i+1}^{-}\right)-k-\xi_{i+1}-\lambda\left|\xi_{i+1}\right| \\
& Y\left(\tau_{i+1}\right)=Y\left(\tau_{i+1}^{-}\right)+\xi_{i+1} .
\end{aligned}
$$

Thus a positive value of $\xi_{i+1}$ indicates that money is being taken from the bank account at time $\tau_{i+1}$ to buy stocks, and conversely if $\xi_{i+1}$ is negative.

If our agent has the amount $x$ on the bank account and $y$ in stocks, his net wealth is given by

$$
H(x, y)=\max \{x+y-\lambda|y|-k, \min \{x, y\}\} .
$$

Therefore it is natural to define the solvency region $\mathcal{S}$ by

$$
\mathcal{S}=\left\{(x, y) \in \mathbf{R}^{2} ; H(x, y) \geq 0\right\}
$$

and we put

$$
\widetilde{\mathcal{S}}=\mathbf{R}^{+} \times \mathcal{S}
$$


Define the line segments $\ell_{1}, \ell_{2}$ by

$$
\begin{aligned}
& \ell_{1}=\{(x, y) ; x+(1-\lambda) y=k, x<0\} \\
& \ell_{2}=\{(x, y) ; x+(1+\lambda) y=k, y<0\}
\end{aligned}
$$

and let the points $P, Q$ be the end points of these segments, i.e.

$$
P=\left(0, \frac{k}{1-\lambda}\right), \quad Q=(k, 0) .
$$

(See Figure 1 and also Remark 2.4.)

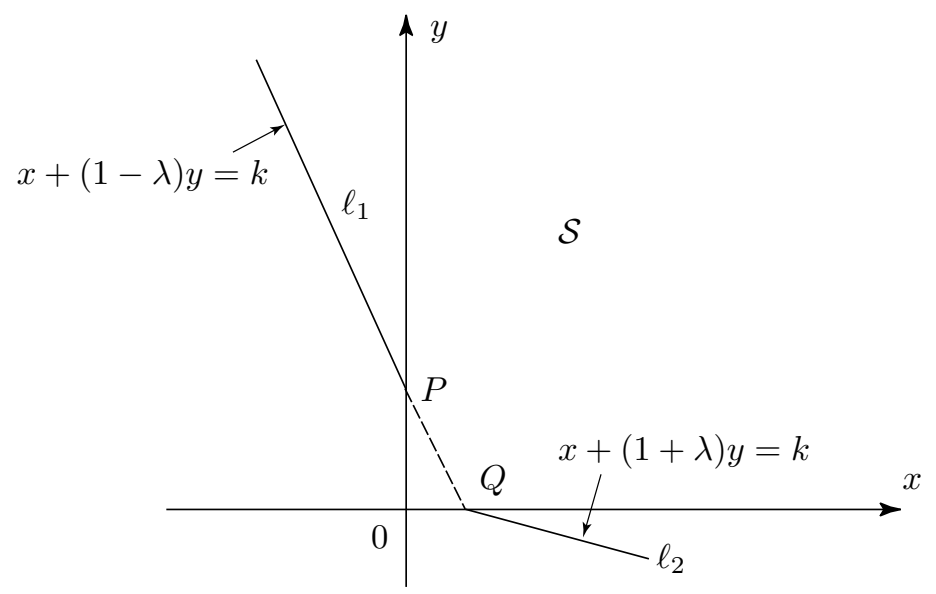

Figure 1

The investor's objective is to maximize over all combined controls $w=(c, v)$ the expression

$$
J^{w}(s, x, y)=E^{s, x, y}\left[\int_{0}^{\infty} e^{-\delta(s+t)} \frac{c^{\gamma}(t)}{\gamma} d t\right]=e^{-\delta s} E^{x, y}\left[\int_{0}^{\infty} e^{-\delta t} \frac{c^{\gamma}(t)}{\gamma} d t\right]
$$

where $\delta>0,0<\gamma<1$ are constants ( $1-\gamma$ is the relative risk aversion coefficient), $E^{s, x, y}$ denotes the expectation with respect to the probability law $P^{s, x, y}$ of

$$
Z(t)=Z^{w}(t):=(s+t, X(t), Y(t)) ; \quad t \geq 0
$$

starting at $z=(s, x, y)$.

We seek the value function(s)

$$
\Phi(s, x, y)=\sup _{w \in \mathcal{W}} J^{w}(s, x, y), \quad \Psi(x, y)=\Phi(0, x, y)
$$


where $\mathcal{W}=\mathcal{W}(x, y)$ is the set of all admissible controls, i.e. all combined controls which satisfy (1.3) and which do not cause the process $Z(t)$ to exit from $\mathcal{S}$. Note that

$$
J^{w}(s, x, y)=e^{-\delta s} J^{w}(0, x, y) \quad \text { and } \quad \Phi(s, x, y)=e^{-\delta s} \Phi(0, x, y)=e^{-\delta s} \Psi(x, y),
$$

so the introduction of the $s$-variable is not really necessary. However, it turns out to be convenient in order to simplify the notation and the arguments in some of the proofs later.

We also seek a corresponding optimal control, i.e. a combined control $w^{*}$ such that

$$
\Phi(s, x, y)=J^{w^{*}}(s, x, y)=e^{-\delta s} \Psi(x, y) .
$$

This problem may be regarded as a generalization of optimal consumption and portfolio problems studied by Merton [M] and Davis \& Norman [DN]. See also Shreve and Soner [SS]. [M] considers the case with no transaction costs $(\lambda=k=0)$, in which case the problem is no longer a combined control problem but a pure stochastic control problem. In this case it is proved in $[\mathrm{M}]$ that it is optimal to choose the portfolio such that

$$
\frac{Y(t)}{X(t)}=\frac{\pi^{*}}{1-\pi^{*}} \quad \text { for all } t
$$

(the Merton line) where

$$
\pi^{*}=\frac{\alpha-r}{(1-\gamma) \sigma^{2}}
$$

Moreover, the corresponding value function in the Merton case $\lambda=k=0$ is given by

$$
\Psi_{0}(x, y)=C_{1}(x+y)^{\gamma}
$$

where

$$
C_{1}=\frac{1}{\gamma} C_{0}^{\gamma-1}, \quad \text { with } \quad C_{0}=\frac{1}{1-\gamma}\left[\delta-\gamma r-\frac{\gamma(\alpha-r)^{2}}{2 \sigma^{2}(1-\gamma)}\right]
$$

provided that

$$
\delta>\gamma\left[r+\frac{(\alpha-r)^{2}}{2 \sigma^{2}(1-\gamma)}\right]
$$

See e.g. [DN, Section 2].

From now on we assume that (1.23) holds.

It is easy to see that

$$
\Psi(x, y) \leq \Psi_{0}(x, y) .
$$

This is also pointed out in Corollary 2.2, to be proved later.

$[\mathrm{DN}]$ and $[\mathrm{SS}]$ consider the case with proportional transaction costs only $(k=0)$, in which case the problem can be formulated as a singular stochastic control problem. It is proved in $[\mathrm{DN}]$ and $[\mathrm{SS}]$ that under some conditions there exist two straight lines $\Gamma_{1}, \Gamma_{2}$ 
through the origin, bounding a cone $N T$, such that it is optimal to make no transactions if $(X(t), Y(t)) \in N T$ and make transactions corresponding to local time at $\partial(N T)$, resulting in reflections back to $N T$ every time $(X(t), Y(t)) \in \partial(N T)$. Depending on the parameters the Merton line may or may not go between the lines $\Gamma_{1}, \Gamma_{2}$ (see the discussion in $[A M S$, Section 7.2]). For an extension of these results to markets with jumps see [FØS1] and [FØS2].

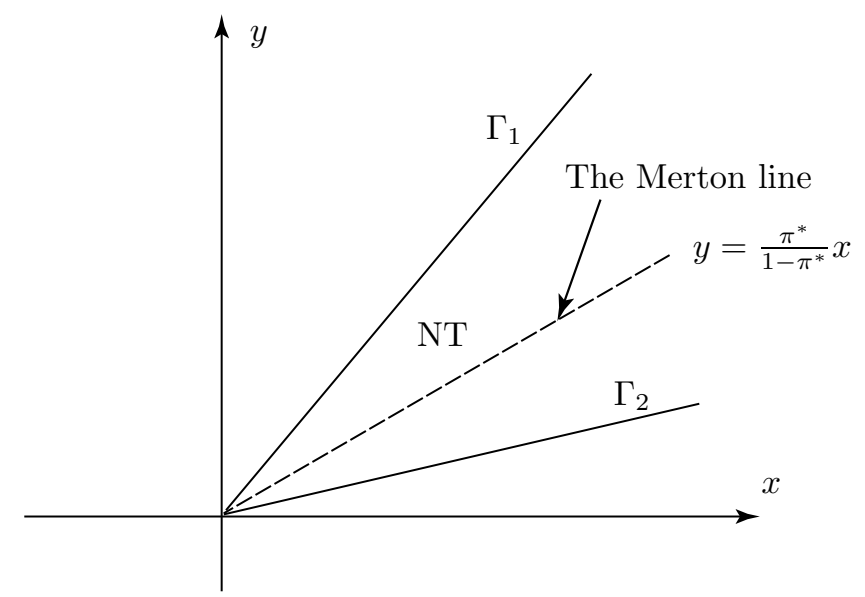

Figure 2

The first paper to model markets with fixed transaction costs $k>0$ by impulse control theory seems to be $[\mathrm{EH}]$, but they do not consider optimal consumption.

Perhaps the paper which is closest to ours is $[\mathrm{K}]$. Here optimal consumption in markets with fixed transaction costs are considered, but consumption is only allowed at the discrete times of the transactions. This makes it possible to put the problem within the framework of impulse control and quasi-variational inequalities.

In our paper we allow consumption to take place at any time, independent of the (discrete) times chosen for the transactions. As explained above we model this as a combined stochastic control and impulse control problem, or a combined control problem, for short.

In Section 2 we introduce quasi-variational Hamilton-Jacobi-Bellman inequalities (QVHJBI) associated to this combined control problem. We point out that if a function $\psi(x, y)$ satisfies these QVHJBI (and some additional smoothness conditions), then $\psi$ coincides with the value function $\Psi$, defined by (1.16). (See Theorem 2.1).

In Section 3 we prove that the value function $\Psi$ is the unique viscosity solution of the QVHJBI formulated in Section 2.

Finally in Section 4 we present some numerical estimates for $\Psi$ and the optimal consumption-investment policy $w^{*}=\left(c^{*}, v^{*}\right)$.

For other recent papers on impulse control and combined control see e.g. [BØ], [MØ], [CZ1], [CZ2] and [BP] and the references therein. We refer to [BL] for a comprehensive treatment of the general theory of impulse control and their quasi-variational inequalities. 
Remark 1.1 Another natural choice of solvency region would be the set

$$
\mathcal{S}_{+}:=[0, \infty) \times[0, \infty)
$$

This choice models a situation where no borrowing or shortselling is allowed. We will mostly use the choice $\mathcal{S}$ given by (1.9) in this paper, but we point out that the proofs carry over to the $\mathcal{S}_{+}$case with only minor modifications. (Usually the $\mathcal{S}_{+}$case is simpler.)

\section{Quasi-variational Hamilton-Jacobi-Bellman inequalities (QVHJBI)}

Let $A^{c}$ be the generator of the process $Z^{c}(t)=\left(s+t, X^{c}(t), Y^{c}(t)\right)$ when there are no transactions, i.e. $A^{c}$ is the partial differential operator given by

$$
\left(A^{c} f\right)(s, x, y)=\frac{\partial f}{\partial s}+(r x-c) \frac{\partial f}{\partial x}+\alpha y \frac{\partial f}{\partial y}+\frac{1}{2} \sigma^{2} y^{2} \frac{\partial^{2} f}{\partial y^{2}}
$$

for any $f: \mathbf{R}^{3} \rightarrow \mathbf{R}$ and $(s, x, y)$ such that the derivatives exist. In particular, if $f(s, x, y)=e^{-\delta s} g(x, y)$ then

$$
\left(A^{c} f\right)(s, x, y)=e^{-\delta s} L^{c} g(x, y)
$$

where

$$
L^{c} g(x, y)=-\delta g+(r x-c) \frac{\partial g}{\partial x}+\alpha y \frac{\partial g}{\partial y}+\frac{1}{2} \sigma^{2} y^{2} \frac{\partial^{2} g}{\partial y^{2}} .
$$

For $(x, y) \in \mathcal{S}$ and $\xi \neq 0$ put

$$
x^{\prime}=x^{\prime}(\xi)=x-k-\xi-\lambda|\xi|, \quad y^{\prime}=y^{\prime}(\xi)=y+\xi .
$$

We define the intervention operator $\mathcal{M}$ by

$$
\mathcal{M} h(x, y)=\sup \left\{h\left(x^{\prime}, y^{\prime}\right) ; \xi \in \mathbf{R} \backslash\{0\},\left(x^{\prime}, y^{\prime}\right) \in \mathcal{S}\right\}
$$

for all locally bounded $h: \mathcal{S} \rightarrow \mathbf{R}^{+},(x, y) \in \mathcal{S}$.

If $\left(x^{\prime}, y^{\prime}\right) \notin \mathcal{S}$ for all $\xi \in \mathbf{R} \backslash\{0\}$ we put $\mathcal{M} h(x, y)=0$. If for all $(x, y) \in \mathcal{S}$ there exists $\left(x^{\prime}, y^{\prime}\right)=\left(x^{\prime}(\xi), y^{\prime}(\xi)\right) \in \mathcal{S}$ such that

$$
\mathcal{M} h(x, y)=h\left(x^{\prime}, y^{\prime}\right)
$$

then we put

$$
\widehat{\xi}(x, y)=\widehat{\xi}_{h}(x, y)=\left(x^{\prime}, y^{\prime}\right)
$$

(More precisely, we let $\widehat{\xi}(x, y)$ denote a measurable selection of the map $(x, y) \rightarrow\left(x^{\prime}, y^{\prime}\right)$.) 
If $\Phi$ is the value function for our problem, then for each $s$ we can interprete $\mathcal{M} \Phi(s, x, y)$ as the maximal value we can obtain by making an admissible transaction at $(s, x, y)$.

Following $[\mathrm{B} \varnothing]$ we call a locally bounded function $h: \widetilde{\mathcal{S}} \rightarrow \mathbf{R}^{+}$stochastically $C^{2}$ with respect to $Z^{c}$ if $\left(A^{c} h\right)(z)$ exists for a.a. $z=(s, x, y)$ with respect to the Green measure (expected occupation time measure) $G\left(z_{0}, \cdot\right)$, and the generalized Dynkin formula holds for $h$, i.e.

$$
E^{z_{0}}\left[h\left(Z^{c}\left(\tau^{\prime}\right)\right)\right]=E^{z_{0}}\left[h\left(Z^{c}(\tau)\right)\right]+E^{z_{0}}\left[\int_{\tau}^{\tau^{\prime}}\left(A^{c} h\right)\left(Z^{c}(t)\right) d t\right]
$$

for all stopping times $\tau, \tau^{\prime}$ such that

$$
\tau \leq \tau^{\prime} \leq T_{R}:=\inf \left\{t>0,\left|Z^{c}(t)\right| \geq R\right\} \wedge R \quad \text { for some } R<\infty .
$$

Recall that for each $z_{0} \in \widetilde{\mathcal{S}}$ the Green measure $G\left(z_{0}, \cdot\right)$ of the process $Z^{c}$ in $\widetilde{\mathcal{S}}$ is defined by

$$
G\left(z_{0}, H\right)=E^{z_{0}}\left[\int_{0}^{\tau} \mathcal{X}_{H}\left(Z^{c}(t)\right) d t\right] \quad \text { for all Borel sets } H \subset \widetilde{\mathcal{S}}
$$

where $\tau=\inf \left\{t>0 ; Z^{c}(t) \notin \widetilde{\mathcal{S}}\right\}$ and $\mathcal{X}_{H}(z)=1$ if $z \in H, \mathcal{X}_{H}(z)=0$ if $z \notin H$.

If $h$ is a function on $\mathcal{S}$ we define

$$
\mathcal{L} h(x, y)=\sup _{c \geq 0}\left\{L^{c} h(x, y)+\frac{c^{\gamma}}{\gamma}\right\} ; \quad(x, y) \in \mathcal{S}
$$

and

$$
\mathcal{L}_{0} h(x, y)=L^{0} h(0, y)=-\delta h+\alpha y \frac{\partial h}{\partial y}+\frac{1}{2} \sigma^{2} y^{2} \frac{\partial^{2} h}{\partial y^{2}}
$$

for all points $(x, y)$ where the partial derivatives of $h$ involved in $L^{c} h$ exist.

We then put (see (1.11)-(1.13) for definitions of $\ell_{1}, \ell_{2}$ and $P$ )

$$
\mathcal{L}_{1} h(x, y)=\left\{\begin{array}{lll}
\mathcal{L} h(x, y) & \text { for } & (x, y) \in \mathcal{S} \backslash\left(\ell_{1} \cup \ell_{2}\right) \backslash[0, P] \\
\mathcal{L}_{0} h(x, y) & \text { for } & (x, y) \in[0, P]
\end{array}\right.
$$

Note that at $[0, P]$ the only admissible consumption is $c=0$.

By adapting Theorem 3.1 in $[\mathrm{B} \varnothing]$ to our situation we get the following sufficient QVHJBI:

Theorem 2.1 Let $\mathcal{S}$ and $\widetilde{\mathcal{S}}$ be as defined in (1.9) and put $U=\mathcal{S} \backslash\left(\ell_{1} \cup \ell_{2}\right), \widetilde{U}=[0, \infty) \times U$.

a) Suppose we can find a locally bounded function $\psi: \mathcal{S} \rightarrow \mathbf{R}^{+}$such that $\psi \in C^{1}(U)$ and

$$
\begin{aligned}
& \phi(s, x, y):=e^{-\delta s} \psi(x, y) \quad \text { is stochastically } C^{2} \text { with respect to } Z^{c}(t) \\
& \text { for all Markov controls } c=c(x, y) \\
& \mathcal{L}_{1} \psi \leq 0 \quad \text { a.e. with respect to } G\left(z_{0}, \cdot\right) \text { on } \widetilde{U} \text { for all } z_{0} \in \widetilde{U} \\
& \psi(x, y) \geq \mathcal{M} \psi(x, y) \quad \text { for all }(x, y) \in U .
\end{aligned}
$$


Then

$$
\psi(x, y) \geq \Psi(x, y) \quad \text { for all }(s, x, y) \in \widetilde{U}
$$

b) Define the continuation region

$$
D=\{(x, y) \in U ; \psi(x, y)>\mathcal{M} \psi(x, y)\} .
$$

Suppose

$$
\mathcal{L}_{1} \psi(x, y)=0 \quad \text { on } D .
$$

and that $\widehat{\xi}(x, y)=\widehat{\xi}_{\psi}(x, y)$ (defined in (2.5)) exists for all $(x, y) \in \mathcal{S}$. Define

$$
c^{*}(x, y)=\left\{\begin{array}{lll}
\left(\frac{\partial \psi}{\partial x}\right)^{\frac{1}{\gamma-1}} & \text { for } & (x, y) \in U \backslash[0, P] \\
0 & \text { for } & (x, y) \in[0, P]
\end{array}\right.
$$

and define the impulse control

$$
v^{*}:=\left(\tau_{1}^{*}, \tau_{2}^{*}, \ldots ; \xi_{1}^{*}, \xi_{2}^{*}, \ldots\right)
$$

as follows:

Put $\tau_{0}^{*}=0$ and inductively

$$
\begin{aligned}
\tau_{k+1}^{*} & =\inf \left\{t>\tau_{k}^{*} ;\left(X^{(k)}(t), Y^{(k)}(t)\right) \notin D\right\} \\
\xi_{k+1}^{*} & =\widehat{\xi}\left(X^{(k)}\left(\tau_{k+1}^{*-}\right), Y^{(k)}\left(\tau_{k+1}^{*-}\right)\right)
\end{aligned}
$$

where $\widehat{\xi}$ is as defined in (2.5) and $\left(X^{(k)}, Y^{(k)}\right)$ is the process obtained by applying the combined control

$$
w^{(k)}:=\left(c^{*},\left(\tau_{1}^{*}, \ldots, \tau_{k}^{*} ; \xi_{1}^{*}, \ldots, \xi_{k}^{*}\right)\right) ; \quad k=1,2, \ldots
$$

Suppose $w^{*}:=\left(c^{*}, v^{*}\right) \in \mathcal{W}$ and that

$$
e^{-\delta t} \psi\left(X^{\left(w^{*}\right)}(t), Y^{\left(w^{*}\right)}(t)\right) \rightarrow 0 \quad \text { as } t \rightarrow \infty \text { a.s. }
$$

and that the family

$$
\left\{e^{-\delta \tau} \psi\left(X^{\left(w^{*}\right)}(\tau), Y^{\left(w^{*}\right)}(\tau)\right) ; \tau \text { stopping time }\right\}
$$

is uniformly integrable. Then

$$
\psi(x, y)=\Psi(x, y)
$$

and $w^{*}$ is optimal. 
Proof. This follows by the proof of Theorem 3.1 in $[\mathrm{B} \varnothing]$ with only minor modifications.

Note that the Hamilton-Jacobi-Bellman inequality (HJBI) (3.7) in [BØ] has the following form in our case, if $(x, y) \in U \backslash[0, P]$,

$$
\mathcal{L} \psi(x, y)=\sup _{c \geq 0}\left\{-\delta \psi+(r x-c) \frac{\partial \psi}{\partial x}+\alpha y \frac{\partial \psi}{\partial y}+\frac{1}{2} \sigma^{2} y^{2} \frac{\partial^{2} \psi}{\partial y^{2}}+\frac{c^{\gamma}}{\gamma}\right\} \leq 0 .
$$

This can only hold if $\frac{\partial \psi}{\partial x}>0$ and then the supremum of this expression is obtained when

$$
c=c^{*}=\left(\frac{\partial \psi}{\partial x}\right)^{\frac{1}{\gamma-1}}
$$

If $(x, y) \in[0, P]$ then only the zero consumption $c=c^{*}=0$ is admissible so again by the HJBI we get $L^{0} \psi(0, y)=0$.

We can use this to prove the claim (1.24):

\section{Corollary 2.2}

a) As in (1.21)-(1.22) let

$$
\Psi_{0}(x, y)=C_{1}(x+y)^{\gamma}
$$

be the value function for the Merton problem $(k=\lambda=0)$. Then

$$
\Psi(x, y) \leq \Psi_{0}(x, y) \quad \text { for all }(x, y) \in \mathcal{S} .
$$

b) Let $b$ be a constant such that

$$
1-\lambda \leq b \leq 1+\lambda
$$

Suppose

$$
\delta>\gamma \alpha
$$

Then there exists $K<\infty$ such that

$$
\Psi(x, y) \leq K(x+b y)^{\gamma} \quad \text { for all }(x, y) \in \mathcal{S} .
$$

Proof. a) We verify that $\psi:=\Psi_{0}$ satisfies the conditions of Theorem 2.1 a): First, $\phi(s, x, y)=e^{-\delta s} \psi(x, y)$ is $C^{2}$ and therefore trivially stochastically $C^{2}$. Hence (2.10) holds. Second, $\psi$ satisfies $(2.11)$, because $\Psi_{0}$ satisfies the Hamilton-Jacobi-Bellman equation. Third, if we put, as in (2.3)

$$
x^{\prime}=x^{\prime}(\xi)=x-\xi-k-\lambda|\xi| \quad \text { and } \quad y^{\prime}=y^{\prime}(\xi)=y+\xi,
$$

then $x^{\prime}+y^{\prime} \leq x+y$ for all $x, y, \xi$ and therefore

$$
\begin{gathered}
\mathcal{M} \Psi_{0}(x, y)=\sup _{\xi \neq 0} \Psi_{0}\left(x^{\prime}, y^{\prime}\right)=\sup _{\xi \neq 0}\left\{C_{1}\left(x^{\prime}+y^{\prime}\right)^{\gamma}\right\} \\
\leq C_{1}(x+y)^{\gamma}=\Psi_{0}(x, y),
\end{gathered}
$$


where $C_{1}$ is defined in (1.22). Therefore (2.12) also holds. Hence (2.21) follows.

b) We proceed as in a), except now we choose $K<\infty$ and define

$$
u(x, y)=K(x+b y)^{\gamma} .
$$

Now we get

$$
x^{\prime}+b y^{\prime}= \begin{cases}x+b y-k-\xi(1+\lambda-b) & \text { for } \xi>0 \\ x+b y-k-\xi(1-\lambda-b) & \text { for } \xi<0\end{cases}
$$

Thus in any case we have, by $(2.22)$,

$$
x^{\prime}+b y^{\prime} \leq x+b y
$$

and this proves that

$$
u(x, y) \geq \mathcal{M} u(x, y) .
$$

It remains to verify that $\psi:=u$ satisfies (2.11): By (2.26) we get

$$
\begin{gathered}
\mathcal{L} u(x, y)=(x+b y)^{\gamma-2}\left[\left(\frac{1-\gamma}{\gamma}(K \gamma)^{\frac{\gamma}{\gamma-1}}-\delta K\right)(x+b y)^{2}\right. \\
\left.+K \gamma(r x+\alpha b y)(x+b y)-\frac{1}{2} \sigma^{2} K \gamma(1-\gamma) b^{2} y^{2}\right] .
\end{gathered}
$$

Hence $\mathcal{L} u(x, y) \leq 0$ for all $(x, y) \in \mathcal{S}$ if and only if

$$
\left[\frac{1-\gamma}{\gamma}(K \gamma)^{\frac{\gamma}{\gamma-1}}-\delta K+K \gamma \alpha\right](x+b y)^{2} \leq \frac{1}{2} \sigma^{2} K \gamma(1-\gamma) b^{2} y^{2}
$$

for all $(x, y) \in \mathcal{S}$. This holds if and only if

$$
\delta>\gamma \alpha+(1-\gamma)(K \gamma)^{\frac{1}{\gamma-1}}
$$

If (2.23) holds, then (2.27) holds for $K$ large enough. Thus (2.24) follows from Theorem 2.1a).

Remark 2.3 Corollary 2.2 proves in particular that the value function $\Psi$ is finite. Moreover, $\Psi(x, y)$ is bounded on every straight line in $\mathcal{S}$ of the form

$$
x+b y=\text { constant }
$$

for every constant $b \in[1-\lambda, 1+\lambda]$. 


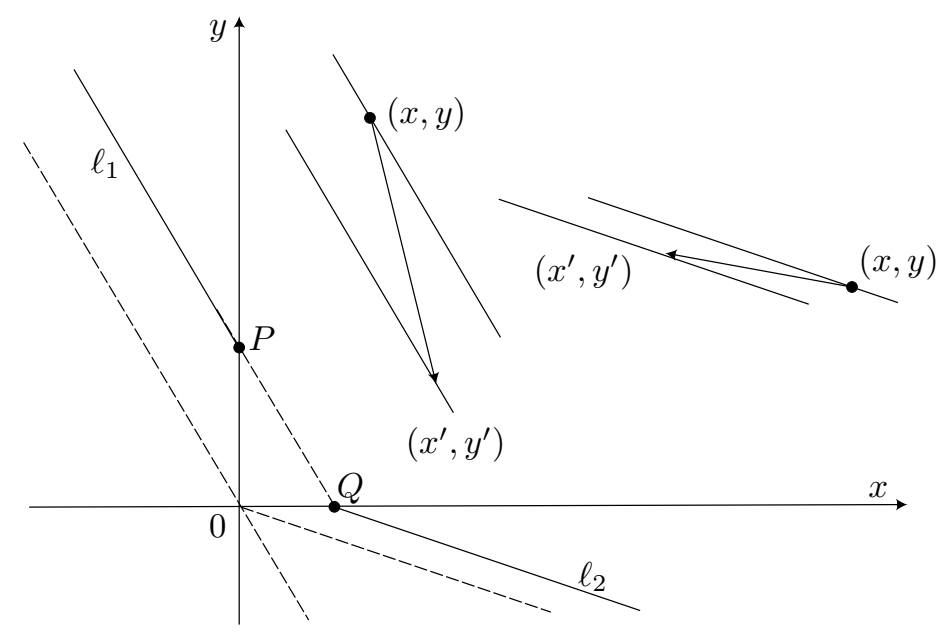

Figure 3

Remark 2.4 (Some comments on the boundary behaviour) Suppose the current position of the investor is a point $(x, y) \in \mathcal{S}$. If we make a transaction of size $\xi$ at that instant, then after the transaction the new position is given by

$$
\left\{\begin{array}{l}
x^{\prime}=x-\xi-\lambda|\xi|-k \\
y^{\prime}=y+\xi
\end{array}\right.
$$

Hence

$$
x^{\prime}+(1-\lambda) y^{\prime}=x+(1-\lambda) y-k-\lambda(|\xi|+\xi)
$$

and

$$
x^{\prime}+(1+\lambda) y^{\prime}=x+(1+\lambda) y-k-\lambda(|\xi|-\xi) .
$$

In particular, if we sell stocks $(\xi<0)$ then $x^{\prime}+(1-\lambda) y^{\prime}=x+(1-\lambda) y-k$, so $(x, y)$ will move to a point $\left(x^{\prime}, y^{\prime}\right)$ on the line parallell to $\ell_{1}$ lying $\frac{k}{1-\lambda}$ units below the parallell of $\ell_{1}$ through $(x, y)$. See Figure 3 .

Similarly, if we buy stocks $(\xi>0)$ then $x^{\prime}+(1+\lambda) y^{\prime}=x+(1+\lambda) y-k$, so $(x, y)$ will move to a point $\left(x^{\prime}, y^{\prime}\right)$ on the line parallell to $\ell_{2}$ lying $\frac{k}{1+\lambda}$ units below the parallell of $\ell_{2}$ through $(x, y)$.

We now use this to deduce the boundary behaviour of the value function $\Psi$ on $\partial \mathcal{S}$.

(i) If $(x, y) \in \ell_{1}$ then we have to make an immediate transaction to avoid that the diffusion $Y(t)$ will take us out of $\mathcal{S}$. By the above we see that the only possibility is to sell stocks of such an amount that $\left(x^{\prime}, y^{\prime}\right)=(0,0)$. We conclude that

$$
\Psi(x, y)=\mathcal{M} \Psi(x, y)=0 \quad \text { for }(x, y) \in \ell_{1} .
$$

(ii) If $(x, y) \in \ell_{2}$ we argue similarly: The only admissible action is to buy stocks immediately of such an amount that $\left(x^{\prime}, y^{\prime}\right)=(0,0)$. Hence 


$$
\Psi(x, y)=\mathcal{M} \Psi(x, y)=0 \quad \text { for }(x, y) \in \ell_{2} .
$$

(iii) On the segment $0<x<k, y=0$ we are not allowed to make any transaction. There is no diffusion and all we can do is to consume optimally. Hence the HJB equation indicates that, with $\mathcal{L}$ as in $(2.7)$ we should have

$$
\mathcal{L} \Psi=-\delta \Psi+r x \frac{\partial \Psi}{\partial x}+\frac{1-\gamma}{\gamma}\left(\frac{\partial \Psi}{\partial x}\right)^{\frac{\gamma}{\gamma-1}}=0 \quad \text { for } x \in(0, k)
$$

provided that $\Psi$ is smooth enough (see Section 4).

(iv) On the segment $x=0,0<y<\frac{k}{1-\lambda}$ we cannot consume because this would bring us outside $\mathcal{S}$. Hence the HJB equation indicates that

$$
\mathcal{L} \Psi(0, y)=c^{*}=0 \quad \text { for } 0<y<\frac{k}{1-\lambda}
$$

and hence that

$$
\mathcal{L}_{0} \Psi:=-\delta \Psi+\alpha y \frac{\partial \Psi}{\partial y}+\frac{1}{2} \sigma^{2} y^{2} \frac{\partial^{2} \Psi}{\partial y^{2}}=0 \quad \text { for } \quad 0<y<\frac{k}{1-\lambda},
$$

provided that $\Psi$ is smooth enough (see Section 4).

Summarizing we see that the boundary behaviour of $\Psi$ on $\partial \mathcal{S}$ can be described by

$$
\begin{cases}\Psi(x, y)=\mathcal{M} \Psi(x, y)=0 & \text { for }(x, y) \in \ell_{1} \cup \ell_{2} \\ \mathcal{L} \Psi(x, y)=0 & \text { for } 0 \leq x \leq k, y=0 \text { i.e., }(x, y) \in[0, Q] \\ \mathcal{L}_{0} \Psi(x, y)=0 & \text { for } x=0,0 \leq y \leq y_{1}=\frac{k}{1-\lambda} \text { i.e., }(x, y) \in[0, P]\end{cases}
$$

Note that $\Psi$ is not continuous on $\partial S$ : The points $\left(0, \frac{k}{1-\lambda}\right)$ and $(k, 0)$ are points of discontinuity. However, $\Psi$ is upper semicontinuous.

\section{$3 \quad$ Viscosity solutions}

Theorem 2.1 is a verification theorem, stating that if we can find a smooth enough function satisfying the required (quasi-) variational inequalities, then we have also found the value function of the problem. It is natural to ask if the converse is also true: Is the value function always a solution of the corresponding (quasi-) variational inequalities? The problem is that the value function need not be smooth enough for these inequalities to be welldefined in the strong sense. In fact, the value function is not even continuous at the points $P$ and $Q$ (see (2.36) and below). However, we shall see that the inequalities are satisfied in a weak sense: The value function is a viscosity solution of the (quasi-) variational inequalities.

We first recall the following concepts, which will be useful for us: 
Definition 3.1 If $C$ is a topological space and $u: C \rightarrow \mathbf{R}$ is a function, then the upper semi-continuous (usc) envelope $\bar{u}: C \rightarrow \mathbf{R}$ and the lower semi-continuous (lsc) envelope $\underline{u}: C \rightarrow \mathbf{R}$ of $u$ are defined by

$$
\bar{u}(x)=\limsup _{\substack{y \rightarrow x \\ y \in C}} u(y), \quad \underline{u}(x)=\liminf _{\substack{y \rightarrow x \\ y \in C}} u(y), \quad \text { respectively. }
$$

We let $\operatorname{USC}(C)$ and $\operatorname{LSC}(C)$ denote the set of usc functions and lsc functions on $C$, respectively.

Note that in general we have

$$
\underline{u} \leq u \leq \bar{u}
$$

and that $u$ is usc if and only if $u=\bar{u}, u$ is lsc if and only if $u=\underline{u}$. In particular, $u$ is continuous if and only if

$$
\underline{u}=u=\bar{u} .
$$

We establish some auxiliary results about the operator $\mathcal{M}$ :

\section{Lemma 3.2}

(i) If $u: \mathcal{S} \rightarrow \mathbf{R}$ is usc, then $\mathcal{M} u$ is usc.

(ii) If $u: \mathcal{S} \rightarrow \mathbf{R}$ is continuous, then $\mathcal{M} u$ is continuous.

Proof. (i) Suppose $u: \mathcal{S} \rightarrow \mathbf{R}$ is usc. For $\zeta=(x, y) \in \mathcal{S}$ define

$$
\ell(\zeta)=\ell(x, y)=\left\{\left(x^{\prime}(\xi), y^{\prime}(\xi)\right) \in \mathcal{S} ; \quad \xi \in \mathbf{R} \backslash\{0\}\right\}
$$

where $x^{\prime}, y^{\prime}$ are as in (2.3). Then $\ell(\zeta)$ is a union of two closed finite line segments and since $u$ is usc there exists $\zeta^{*} \in \ell(\zeta)$ such that

$$
\mathcal{M} u(\zeta)=\sup \left\{u\left(\zeta^{\prime}\right) ; \zeta^{\prime} \in \ell(\zeta)\right\}=u\left(\zeta^{*}\right) .
$$

Fix $\zeta_{0} \in \mathcal{S}$ and let $\left\{\zeta_{n}\right\}_{n=1}^{\infty}$ be a sequence in $\mathcal{S}$ such that $\zeta_{n} \rightarrow \zeta_{0}$ as $n \rightarrow \infty$. We must show that

$$
\mathcal{M} u\left(\zeta_{0}\right) \geq \limsup _{n \rightarrow \infty} \mathcal{M} u\left(\zeta_{n}\right) .
$$

Let $\hat{\zeta}$ be a cluster point of $\left\{\zeta_{n}^{*}\right\}_{n=1}^{\infty}$, i.e. $\hat{\zeta}$ is the limit of some convergent subsequence $\left\{\zeta_{n_{k}}^{*}\right\}_{k=1}^{\infty}$ of $\left\{\zeta_{n}^{*}\right\}_{n=1}^{\infty}$. Since $\zeta_{n} \rightarrow \zeta_{0}$ we see that $\ell\left(\zeta_{n}\right) \rightarrow \ell\left(\zeta_{0}\right)$, in the natural sense. Hence, since $\zeta_{n_{k}}^{*} \in \ell\left(\zeta_{n_{k}}\right)$ for all $k$, we conclude that $\hat{\zeta}=\lim _{k \rightarrow \infty} \zeta_{n_{k}}^{*} \in \ell\left(\zeta_{0}\right)$. Therefore

$$
\mathcal{M} u\left(\zeta_{0}\right) \geq u(\hat{\zeta}) \geq \limsup _{n \rightarrow \infty} u\left(\zeta_{n}^{*}\right)=\limsup _{n \rightarrow \infty} \mathcal{M} u\left(\zeta_{n}\right) .
$$

(ii) Suppose $u: \mathcal{S} \rightarrow \mathbf{R}$ is continuous. Fix $\zeta_{0} \in \mathcal{S}$ and let $\zeta_{n} \rightarrow \zeta_{0}$ as in (i). By (i) it suffices to show that

$$
\mathcal{M} u\left(\zeta_{0}\right) \leq \liminf _{n \rightarrow \infty} \mathcal{M} u\left(\zeta_{n}\right)
$$


Suppose not. Then $u\left(\zeta_{0}^{*}\right)=\mathcal{M} u\left(\zeta_{0}\right)>\liminf _{n \rightarrow \infty} \mathcal{M} u\left(\zeta_{n}\right)+\varepsilon=\liminf _{n \rightarrow \infty} u\left(\zeta_{n}^{*}\right)+\varepsilon$ for some $\varepsilon>0$.

Since $u$ is continuous there is a neighbourhood $G$ of $\zeta_{0}^{*}$ such that

$$
u\left(\zeta^{\prime}\right) \geq \liminf u\left(\zeta_{n}^{*}\right)+\varepsilon \quad \text { for all } \zeta^{\prime} \in G .
$$

But if $n$ is big enough we have $\ell\left(\zeta_{n}\right) \cap G \neq \emptyset$ so since $\zeta_{n}^{*}$ is a maximum point of $u$ on $\ell\left(\zeta_{n}\right)$ we have

$$
u\left(\zeta_{n}^{*}\right) \geq u\left(\zeta^{\prime}\right) \quad \text { for } n \text { big enough . }
$$

This contradiction shows that $(*)$ holds, and the proof is complete.

Lemma 3.3 (i) Let $u: \mathcal{S} \rightarrow \mathbf{R}$. Then $\overline{\mathcal{M} u} \leq \mathcal{M} \bar{u}$.

(ii) Let $\psi: \mathcal{S} \rightarrow \mathbf{R}$ be such that $\psi \geq \mathcal{M} \psi$. Then $\underline{\psi} \geq \mathcal{M} \underline{\psi}$.

Proof. (i): Choose $\zeta_{0}, \zeta_{n} \in \mathcal{S}, n=1,2, \ldots$, such that $\zeta_{n} \rightarrow \zeta_{0}$ and $\mathcal{M} u\left(\zeta_{n}\right) \rightarrow \overline{\mathcal{M} u}\left(\zeta_{0}\right)$ as $n \rightarrow \infty$. Then by Lemma 3.2 (i) applied to the usc function $\bar{u}$

$$
\overline{\mathcal{M} u}\left(\zeta_{0}\right)=\lim _{n \rightarrow \infty} \mathcal{M} u\left(\zeta_{n}\right) \leq \limsup _{n \rightarrow \infty} \mathcal{M} \bar{u}\left(\zeta_{n}\right) \leq \mathcal{M} \bar{u}\left(\zeta_{0}\right)
$$

(ii): Choose $\zeta_{0}, \zeta_{n} \in \mathcal{S}, n=1,2, \ldots$, such that $\zeta_{n} \rightarrow \zeta_{0}$ and $\mathcal{M} \psi\left(\zeta_{n}\right) \rightarrow \underline{\mathcal{M} \psi}\left(\zeta_{0}\right)$ as $n \rightarrow \infty$. Then

$$
\underline{\psi}\left(\zeta_{0}\right) \geq \underline{\mathcal{M} \psi}\left(\zeta_{0}\right)=\lim _{n \rightarrow \infty} \mathcal{M} \psi\left(\zeta_{n}\right) \geq \liminf _{n \rightarrow \infty} \mathcal{M} \underline{\psi}\left(\zeta_{n}\right) \geq \mathcal{M} \underline{\psi}\left(\zeta_{0}\right)
$$

Corollary 3.4 Suppose $u: \mathcal{S} \rightarrow \mathbf{R}$ is usc and $u\left(\zeta_{0}\right)>\mathcal{M} u\left(\zeta_{0}\right)+\eta$ for some $\zeta_{0} \in \mathcal{S}$, $\eta>0$. Then $u\left(\zeta_{0}\right)>\overline{\mathcal{M} u}\left(\zeta_{0}\right)+\eta$.

Proof. $u\left(\zeta_{0}\right)>\mathcal{M} u\left(\zeta_{0}\right)+\eta=\mathcal{M} \bar{u}\left(\zeta_{0}\right) \geq \overline{\mathcal{M} u}\left(\zeta_{0}\right)+\eta$ by Lemma 3.3 (i).

As in (2.7) we let $\mathcal{L}$ be the differential operator

$$
\mathcal{L} h(x, y)=\sup _{c \geq 0}\left\{-\delta h+(r x-c) \frac{\partial h}{\partial x}+\alpha y \frac{\partial h}{\partial y}+\frac{1}{2} \sigma^{2} y^{2} \frac{\partial^{2} h}{\partial y^{2}}+\frac{c^{\gamma}}{\gamma}\right\}
$$

and as in (2.2) we put

$$
\mathcal{M} h(x, y)=\sup _{\xi \neq 0}\left\{h\left(x^{\prime}, y^{\prime}\right) ;\left(x^{\prime}, y^{\prime}\right) \in \mathcal{S}\right\} \quad \text { for } \quad(x, y) \in \mathcal{S},
$$

where

$$
x^{\prime}=x-k-\xi-\lambda|\xi|, \quad y^{\prime}=y+\xi .
$$


The inequalities (2.11), (2.12) and (2.13) of Theorem 2.1 together with the boundary behaviour (2.36) can be combined into one equation as follows:

$$
F\left(D^{2} \Psi(\zeta), D \Psi(\zeta), \Psi, \zeta\right)=0 \quad \text { for all } \zeta=(x, y) \in \mathcal{S},
$$

where

$$
F: \mathbf{R}^{2 \times 2} \times \mathbf{R}^{2} \times \mathbf{R}^{\mathcal{S}} \times \mathbf{R}^{2} \rightarrow \mathbf{R}
$$

is defined by

$$
F(A, p, g, \zeta)= \begin{cases}\max \{\Lambda(A, p, g, \zeta),(\mathcal{M} g-g)(\zeta)\} ; & \zeta \in \mathcal{S}^{0} \\ \Lambda(A, p, g, \zeta) ; & \zeta \in[0, Q] \\ \Lambda_{0}(A, p, g, \zeta) ; & \zeta \in[0, P] \\ (\mathcal{M} g-g)(\zeta) ; & \zeta \in \ell_{1} \cup \ell_{2}\end{cases}
$$

where

$$
\Lambda(A, p, g, \zeta)=-\delta g+r \zeta_{1} p_{1}+\alpha \zeta_{2} p_{2}+\frac{1}{2} \sigma^{2} \zeta_{2}^{2} A_{22}+\max _{c \geq 0}\left(-c p_{1}+\frac{c^{\gamma}}{\gamma}\right)
$$

and

$$
\Lambda_{0}(A, p, g, \zeta)=-\delta g+\alpha \zeta_{2} p_{2}+\frac{1}{2} \sigma^{2} \zeta_{2}^{2} A_{22}, \quad A=\left[A_{i j}\right]_{1 \leq i, j \leq 2} .
$$

Note that $F$ is not a local operator: The value of $F$ at $(A, p, g, \zeta)$ depends on the value of $g$ on the whole space $\mathcal{S}$.

Also note that

$$
\bar{F}(A, p, g, \zeta)=\max \{\Lambda(A, p, g, \zeta),(\mathcal{M} g-g)(\zeta)\} \text { for all } \zeta \in \mathcal{S}
$$

and that

$$
\underline{F}(A, p, g, \zeta)=F(A, p, g, \zeta) \quad \text { (i.e. } F \text { is lsc) }
$$

Following Barles $[\mathrm{B}]$ we now give the definition of viscosity solution of elliptic equations of type (3.4):

\section{Definition 3.5}

a) A function $u \in \operatorname{USC}(\mathcal{S})$ is a viscosity subsolution of

$$
F\left(D^{2} u(\zeta), D u(\zeta), u, \zeta\right)=0 \quad \text { for all } \zeta=(x, y) \in \mathcal{S}
$$

if for every function $f$ which is $C^{2}$ in a neighbourhood of $\mathcal{S}$ and for every point $\zeta_{0} \in \mathcal{S}$ such that $f \geq u$ on $\mathcal{S}$ and $f\left(\zeta_{0}\right)=u\left(\zeta_{0}\right)$ we have

$$
\bar{F}\left(D^{2} f\left(\zeta_{0}\right), D f\left(\zeta_{0}\right), u, \zeta_{0}\right) \geq 0 .
$$


b) A function $u \in \operatorname{LSC}(\mathcal{S})$ is a viscosity supersolution of (3.10) if for every function $f$ which is $C^{2}$ in a neighbourhood of $\mathcal{S}$ and for every point $\zeta_{0} \in \mathcal{S}$ such that $f \leq u$ on $\mathcal{S}$ and $f\left(\zeta_{0}\right)=u\left(\zeta_{0}\right)$ we have

$$
\underline{F}\left(D^{2} f\left(\zeta_{0}\right), D f\left(\zeta_{0}\right), u, \zeta_{0}\right) \leq 0 .
$$

c) We say that a function $u: \mathcal{S} \rightarrow \mathbf{R}$ is a viscosity solution of (3.10) if $u$ is locally bounded and $\bar{u}$ is a viscosity subsolution and $\underline{u}$ is a viscosity supersolution of (3.10).

An equivalent definition of viscosity solutions which is useful for proving uniqueness results is the following (see [CIL, Section 2]):

\section{Definition 3.6}

a) A function $u \in \operatorname{USC}(\mathcal{S})$ is a viscosity subsolution of (3.4) if

$$
\bar{F}(A, p, u, \zeta) \geq 0 \quad \text { for all }(p, A) \in \bar{J}_{\mathcal{S}}^{2,+} u(\zeta), \zeta \in \mathcal{S}
$$

b) A function $u \in \operatorname{LSC}(\mathcal{S})$ is a viscosity supersolution of (3.4) if

$$
\underline{F}(A, p, u, \zeta) \leq 0 \quad \text { for all }(p, A) \in \bar{J}_{\mathcal{S}}^{2,-} u(\zeta), \zeta \in \mathcal{S} .
$$

Here the second order "superjets" $J_{\mathcal{S}}^{2,+}, J_{\mathcal{S}}^{2,-}$ and their "closures" $\bar{J}_{\mathcal{S}}^{2,+}, \bar{J}_{\mathcal{S}}^{2,-}$ are defined by

$$
\begin{aligned}
& J_{\mathcal{S}}^{2,+} u(\zeta)=\left\{(p, A) \in \mathbf{R}^{2} \times \mathbf{R}^{2 \times 2}\right. \\
& \left.\quad \limsup _{\substack{\eta \rightarrow \zeta \\
\eta \in \mathcal{S}}}\left\{\left[u(\eta)-u(\zeta)-p \cdot(\eta-\zeta)-\frac{1}{2}(\eta-\zeta)^{T} A(\eta-\zeta)\right]|\eta-\zeta|^{-2}\right\} \leq 0\right\}
\end{aligned}
$$

(where ()$^{T}$ denotes matrix transposed)

$$
\bar{J}_{\mathcal{S}}^{2,+} u(\zeta)=\left\{(p, A) \in \mathbf{R}^{2} \times \mathbf{R}^{2 \times 2} ; \exists\left(\zeta_{n}, p_{n}, A_{n}\right) \in \mathcal{S} \times \mathbf{R}^{2} \times \mathbf{R}^{2 \times 2},\right.
$$

with $\left(p_{n}, A_{n}\right) \in J_{\mathcal{S}}^{2,+} u\left(\zeta_{n}\right)$ and $\left(\zeta_{n}, u\left(\zeta_{n}\right), p_{n}, A_{n}\right)$

$$
\rightarrow(\zeta, u(\zeta), p, A) \quad \text { when } n \rightarrow \infty\}
$$

and

$$
J_{\mathcal{S}}^{2,-} u=-J_{\mathcal{S}}^{2,+}(-u), \quad \bar{J}_{\mathcal{S}}^{2,-} u=-\bar{J}_{\mathcal{S}}^{2,+}(-u) .
$$

We are now ready for the first main result of this section:

Theorem 3.7 Suppose (2.23) holds. Then the value function $\Psi$ is a viscosity solution of (3.4). 
Proof. We first make some useful observations:

Suppose $w=(c, v) \in \mathcal{W}$ is an admissible control with $v=\left(\tau_{1}, \tau_{2}, \ldots ; \xi_{1}, \xi_{2}, \ldots\right)$ where $\tau_{1}>0$ a.s. Then by the Markov property we have, with $J^{w}$ as in (1.14),

$$
J^{w}(z)=E^{z}\left[\int_{0}^{\tau} e^{-\delta(s+t)} \frac{c^{\gamma}(t)}{\gamma} d t+J^{w}\left(Z^{(w)}(\tau)\right)\right]
$$

for all stopping times $\tau \leq \tau_{1}$.

Note that

$$
\Psi(\zeta) \geq \mathcal{M} \Psi(\zeta) \quad \text { for all } \zeta \in \mathcal{S}
$$

To see this, suppose on the contrary that there exists $\zeta_{1}$ such that

$$
\Psi\left(\zeta_{1}\right)<\mathcal{M} \Psi\left(\zeta_{1}\right)
$$

This would mean that we could improve the performance at $\zeta_{1}$ by making a transaction immediately. This contradicts that $\Psi\left(\zeta_{1}\right)$ is the optimal performance value at $\zeta_{1}$.

Also note that since $\tau_{1}$ is a stopping time, we know that $\left\{\omega ; \tau_{1}(\omega)=0\right\}$ is $\mathcal{F}_{0^{-}}$ measurable and hence this event has either probability 1 or 0 . So we either have

$$
\tau_{1}(\omega)=0 \quad \text { a.s. } \quad \text { or } \quad \tau_{1}(\omega)>0 \quad \text { a.s. }
$$

a) We prove that $\bar{\Psi}$ is a viscosity subsolution. To this end, let $f$ be a $C^{2}$ function in a neighbourhood of $\mathcal{S}$ and let $\zeta_{0} \in \mathcal{S}$ be such that $f \geq \bar{\Psi}$ on $\mathcal{S}$ and $f\left(\zeta_{0}\right)=\bar{\Psi}\left(\zeta_{0}\right)$. We consider two cases separately:

Case 1. $\bar{\Psi}\left(\zeta_{0}\right) \leq \mathcal{M} \bar{\Psi}\left(\zeta_{0}\right)$.

Then by (3.8) $\bar{F}\left(D^{2} f\left(\zeta_{0}\right), D f\left(\zeta_{0}\right), f\left(\zeta_{0}\right), \bar{\Psi}, \zeta_{0}\right) \geq(\mathcal{M} \bar{\Psi}-\bar{\Psi})\left(\zeta_{0}\right)=0$ and hence $(3.11)$ holds at $\zeta_{0}$ for $u=\bar{\Psi}$.

Case 2. $\bar{\Psi}\left(\zeta_{0}\right)>\mathcal{M} \bar{\Psi}\left(\zeta_{0}\right)$.

It suffices to prove that $\mathcal{L} f\left(\zeta_{0}\right) \geq 0$.

We argue by contradiction: Suppose $\zeta_{0}=\left(x_{0}, y_{0}\right) \in \mathcal{S}$ and $\mathcal{L} f\left(\zeta_{0}\right)<0$. Then from the definition $(3.1)$ of $\mathcal{L}$ we deduce that $\frac{\partial f}{\partial x}\left(\zeta_{0}\right)>0$. Hence by continuity, $\frac{\partial f}{\partial x}(\zeta)>0$ in a neighbourhood $G$ of $\zeta_{0}$. But then, with $\zeta=(x, y)$,

$$
\mathcal{L} f(\zeta)=-\delta f(\zeta)+(r x-\widehat{c}) \frac{\partial f}{\partial x}+\alpha y \frac{\partial f}{\partial y}+\frac{1}{2} \sigma^{2} y^{2} \frac{\partial^{2} f}{\partial y^{2}}+\frac{\widehat{c}^{\gamma}}{\gamma}
$$

with $\widehat{c}=\widehat{c}(\zeta)=\left(\frac{\partial f}{\partial x}\right)^{\frac{1}{\gamma-1}}$ for all $\zeta \in G \cap \mathcal{S}$.

Hence $\mathcal{L} f(\zeta)$ is continuous on $G \cap \mathcal{S}$ and so there exists a (bounded) neighbourhood $G_{\rho}$ of $\zeta_{0}$ such that $G_{\rho}=\left\{(x, y) ;\left|x-x_{0}\right|<\rho\right.$ and $\left.\left|y-y_{0}\right|<\rho\right\}$ for some $\rho>0$ and

$$
\mathcal{L} f(\zeta)<\frac{1}{2} \mathcal{L} f\left(\zeta_{0}\right)<0 \quad \text { for all } \zeta \in G_{\rho} \cap \mathcal{S} .
$$


Now let $\eta$ be any number such that

$$
0<\eta<(\bar{\Psi}-\mathcal{M} \bar{\Psi})\left(\zeta_{0}\right)
$$

Since $\bar{\Psi}\left(\zeta_{0}\right)>\mathcal{M} \bar{\Psi}\left(\zeta_{0}\right)+\eta$ we can by Corollary 3.4 find a sequence $\left\{\zeta_{n}\right\}_{n=1}^{\infty} \subset G_{\rho} \cap \mathcal{S}$ such that $\zeta_{n} \rightarrow \zeta_{0}$ and $\Psi\left(\zeta_{n}\right) \rightarrow \bar{\Psi}\left(\zeta_{0}\right)$ as $n \rightarrow \infty$ and

$$
\mathcal{M} \Psi\left(\zeta_{n}\right)<\Psi\left(\zeta_{n}\right)-\eta \quad \text { for all } n \geq 1
$$

Choose $\varepsilon \in(0, \eta)$. Since $\bar{\Psi}\left(\zeta_{0}\right)=f\left(\zeta_{0}\right)$ we can choose $n_{0}$ such that

$$
\left|\Psi\left(\zeta_{n}\right)-f\left(\zeta_{n}\right)\right|<\varepsilon \quad \text { for all } n \geq n_{0} .
$$

In the following we fix $n \geq n_{0}$ and put $\zeta=\zeta_{n}$.

Let $\widetilde{w}=(\widetilde{c}, \widetilde{v})$ with $\widetilde{v}=\left(\widetilde{\tau}_{1}, \widetilde{\tau}_{2}, \ldots ; \widetilde{\xi}_{1}, \widetilde{\xi}_{2}, \ldots\right)$ be an $\varepsilon$-optimal control for $\zeta$, in the sense that

$$
\Psi(\zeta) \leq J^{\tilde{w}}(0, \zeta)+\varepsilon
$$

If $\widetilde{\tau}_{1}=0$ a.s. then $\left(X^{(\tilde{w})}, Y^{(\tilde{w})}\right)$ makes an immediate jump from $\zeta$ to some point $\zeta^{\prime} \in \mathcal{S}$ and hence by (3.17)

$$
J^{\tilde{w}}(0, \zeta)=E^{0, \zeta_{0}}\left[J^{\tilde{w}}\left(0, \zeta^{\prime}\right)\right] .
$$

But then

$$
\Psi(\zeta) \leq J^{\tilde{w}}(0, \zeta)+\varepsilon=E^{0, \zeta}\left[J^{\tilde{w}}\left(0, \zeta^{\prime}\right)\right]+\varepsilon \leq E^{0, \zeta}\left[\Psi\left(\zeta^{\prime}\right)\right]+\varepsilon \leq \mathcal{M} \Psi(\zeta)+\varepsilon
$$

which contradicts (3.21). We conclude that $\widetilde{\tau}_{1}>0$ a.s.

Fix $R<\infty$ and define $\tau$ to be the stopping time

$$
\tau=\tau(\varepsilon)=\widetilde{\tau}_{1} \wedge R \wedge \inf \left\{t>0 ;\left(X^{(\tilde{w})}(t), Y^{(\tilde{w})}(t)\right) \notin G_{\rho}\right\} .
$$

Then by the Dynkin formula we have

$$
\begin{aligned}
& E^{0, \zeta}\left[e^{-\delta \tau} f\left(X^{(\tilde{w})}(\tau), Y^{(\tilde{w})}(\tau)\right)\right]=f(\zeta)+E^{0, \zeta}\left[\int_{0}^{\tau} e^{-\delta t} L^{\tilde{c}} f\left(X^{(\tilde{w})}(t), Y^{(\tilde{w})}(t)\right) d t\right] \\
& \quad+E^{0, \zeta}\left[e^{-\delta \tau}\left[f\left(X^{(\tilde{w})}(\tau), Y^{(\tilde{w})}(\tau)\right)-f\left(X^{(\tilde{w})}\left(\tau^{-}\right), Y^{(\tilde{w})}\left(\tau^{-}\right)\right)\right]\right]
\end{aligned}
$$

or

$$
E^{0, \zeta}\left[e^{-\delta \tau} f\left(X^{(\tilde{w})}\left(\tau^{-}\right), Y^{(\tilde{w})}\left(\tau^{-}\right)\right)\right]=f(\zeta)+E^{0, \zeta}\left[\int_{0}^{\tau} e^{-\delta t} L^{\tilde{c}} f\left(X^{(\tilde{w})}(t), Y^{(\tilde{w})}(t)\right) d t\right]
$$


Combining this with (3.17) we get, since $\Psi \geq \mathcal{M} \Psi$,

$$
\begin{aligned}
& \Psi(\zeta) \leq J^{(\tilde{w})}(0, \zeta)+\varepsilon \\
&=E^{0, \zeta}\left[\int_{0}^{\tau} e^{-\delta t} \frac{\tilde{c}^{\gamma}(t)}{\gamma} d t+J^{\tilde{w}}\left(Z^{(\tilde{w})}(\tau)\right)\right]+\varepsilon \\
& \leq E^{0, \zeta}\left[\int_{0}^{\tau} e^{-\delta t} \frac{\tilde{c}^{\gamma}(t)}{\gamma} d t+e^{-\delta \tau} \Psi\left(X^{(\tilde{w})}(\tau), Y^{(\tilde{w})}(\tau)\right)\right]+\varepsilon \\
& \leq E^{0, \zeta}\left[\int_{0}^{\tau} e^{-\delta t} \frac{\tilde{c}^{\gamma}(t)}{\gamma} d t+e^{-\delta \tau}\left\{\Psi\left(X^{(\tilde{w})}\left(\tau^{-}\right), Y^{(\tilde{w})}\left(\tau^{-}\right)\right) \cdot \chi_{\tau<\tilde{\tau}_{1}}\right.\right. \\
& \leq\left.\left.+\mathcal{M} \Psi\left(X^{(\tilde{w})}\left(\tau^{-}\right), Y^{(\tilde{w})}\left(\tau^{-}\right)\right) \cdot \chi_{\tau=\tilde{\tau}_{1}}\right\}\right]+\varepsilon \\
& \leq\left.\int_{0}^{\tau} e^{-\delta t} \frac{\tilde{c}^{\gamma}(t)}{\gamma} d t+e^{-\delta \tau} \Psi\left(X^{(\tilde{w})}\left(\tau^{-}\right), Y^{(\tilde{w})}\left(\tau^{-}\right)\right)\right]+\varepsilon \\
&=\left.f(\zeta)+E^{-\delta t} \frac{\tilde{c}^{\gamma}(t)}{\gamma} d t+e^{-\delta \tau} f\left(X^{(\tilde{w})}\left(\tau^{-}\right), Y^{(\tilde{w})}\left(\tau^{-}\right)\right)\right]+\varepsilon \\
& \leq \Psi(\zeta)+E^{0, \zeta}\left[\int_{0}^{\tau} e^{-\delta t} \mathcal{L} f\left(X^{(\tilde{w})}(t), Y^{(\tilde{w})}(t)\right) d t\right]+2 \varepsilon .
\end{aligned}
$$

We conclude from this that

$$
E^{0, \zeta}\left[\int_{0}^{\tau} e^{-\delta t} \mathcal{L} f\left(X^{(\tilde{w})}(t), Y^{(\tilde{w})}(t) d t\right] \geq-2 \varepsilon .\right.
$$

On the other hand, from (3.19) we deduce that

$$
E^{0, \zeta}\left[\int_{0}^{\tau} e^{-\delta t} \mathcal{L} f\left(X^{(\tilde{w})}(t), Y^{(\tilde{w})}(t)\right) d t\right] \leq \frac{1}{2 \delta} \mathcal{L} f(\zeta)\left(1-E^{0, \zeta}\left[e^{-\delta \tau}\right]\right) .
$$

We claim that

$$
E^{0, \zeta_{n}}\left[e^{-\delta \tau(\varepsilon)}\right] \text { is bounded away from } 1
$$

when $n \rightarrow \infty$ and $\varepsilon \rightarrow 0$.

If this claim is proved, then we see that (3.23) contradicts (3.24) if $\varepsilon$ is small enough. This contradiction proves that $\mathcal{L} f\left(\zeta_{0}\right) \leq 0$ and hence $(3.11)$ holds. Therefore, to complete the proof we must verify the claim (3.25). 
First note that for $t<\tau$ we have by (1.4)

$$
X^{(\tilde{w})}(t)=X(0) e^{r t}-e^{r t} \int_{0}^{t} e^{-r s} \tilde{c}(s) d s \geq X(0)-\rho
$$

and hence, with some constant $C_{2}<\infty$,

$$
\begin{aligned}
& \int_{0}^{\tau} e^{-\delta t} \frac{\tilde{c}^{\gamma}(t)}{\gamma} d t \leq \frac{1}{\gamma}\left[\int_{0}^{\tau} e^{-r t} \tilde{c}(t) d t\right]^{\gamma}\left[\int_{0}^{\tau} e^{\frac{r \gamma-\delta}{1-\gamma} t} d t\right]^{1-\gamma} \\
& \quad \leq C_{2}\left(X(0)\left(1-e^{-r \tau}\right)+\rho e^{-r \tau}\right)^{\gamma}, \quad \text { since } r \gamma-\delta<0 \text { by (1.2) and (2.23). }
\end{aligned}
$$

Combining this with (3.17) we get

$$
\begin{aligned}
\Psi(\zeta)-\varepsilon & \leq J^{(\tilde{w})}(0, \zeta) \\
\leq & E^{0, \zeta}\left[\int_{0}^{\tau} e^{-\delta t} \frac{\tilde{c}^{\gamma}(t)}{\gamma} d t+e^{-\delta \tau} \Psi\left(X^{(\tilde{w})}(\tau), Y^{(\tilde{w})}(\tau)\right)\right] \\
\leq & E^{0, \zeta}\left[C_{2}\left(x-(x-\rho) e^{-r \tau}\right)^{\gamma}\right]+E^{0, \zeta}\left[e^{-\delta \tau} \Psi\left(X^{(\tilde{w})}\left(\tau^{-}\right), Y^{(\tilde{w})}\left(\tau^{-}\right)\right) \cdot \mathcal{X}_{\tilde{\tau}_{1}>\tau}\right] \\
& +E^{0, \zeta}\left[e^{-\delta \tau}\left\{\Psi\left(X^{(\tilde{w})}(\tau), Y^{(\tilde{w})}(\tau)\right)-\Psi\left(X^{(\tilde{w})}\left(\tau^{-}\right), Y^{(\tilde{w})}\left(\tau^{-}\right)\right)\right\} \cdot \mathcal{X}_{\tilde{\tau}_{1} \leq \tau}\right] \\
\leq & E^{0, \zeta}\left[C_{2}\left(x-(x-\rho) e^{-r \tau}\right)^{\gamma}\right]+E^{0, \zeta}\left[e^{-\delta \tau} \Psi\left(X^{(\tilde{w})}\left(\tau^{-}\right), Y^{(\tilde{w})}\left(\tau^{-}\right)\right) \cdot \mathcal{X}_{\tilde{\tau}_{1}>\tau}\right] \\
& \quad+E^{0, \zeta}\left[e^{-\delta \tau} \mathcal{M} \Psi\left(X^{(\tilde{w})}\left(\tau^{-}\right), Y^{(\tilde{w})}\left(\tau^{-}\right)\right) \cdot \mathcal{X}_{\tilde{\tau}_{1} \leq \tau}\right] \\
\leq & E^{0, \zeta}\left[C_{2}\left(x-(x-\rho) e^{-r \tau}\right)^{\gamma}\right]+E^{0, \zeta}\left[e^{-\delta \tau} \mathcal{X}_{\tilde{\tau}_{1}>\tau}\right] \cdot \sup \left\{\Psi(\tilde{\zeta}) ; \tilde{\zeta} \in G_{\rho}\right\} \\
& \quad+E^{0, \zeta}\left[e^{-\delta \tau} \mathcal{X}_{\tilde{\tau}_{1} \leq \tau}\right] \cdot \sup \left\{\mathcal{M} \Psi(\tilde{\zeta}) ; \tilde{\zeta} \in G_{\rho}\right\} .
\end{aligned}
$$

Now if there exists a sequence $\varepsilon_{k} \rightarrow 0$ and a subsequence $\left\{\zeta_{n_{k}}\right\}$ of $\left\{\zeta_{n}\right\}$ such that

$$
E^{0, \zeta_{n_{k}}}\left[e^{-\delta \tau\left(\varepsilon_{k}\right)}\right] \rightarrow 1 \quad \text { when } k \rightarrow \infty,
$$

then

$$
E^{0, \zeta_{n_{k}}}\left[e^{-\delta \tau\left(\varepsilon_{k}\right)} \mathcal{X}_{\tilde{\tau}_{1}>\tau}\right] \rightarrow 0 \quad \text { when } k \rightarrow \infty,
$$

so by choosing $\zeta=\zeta_{n_{k}}, \tau=\tau\left(\varepsilon_{k}\right)$ in (3.26) and letting $k \rightarrow \infty$ we obtain

$$
\bar{\Psi}\left(\zeta_{0}\right) \leq C_{2} \rho^{\gamma}+\sup \left\{\mathcal{M} \Psi(\tilde{\zeta}) ; \tilde{\zeta} \in G_{\rho}\right\}
$$

Hence by Lemma 3.3 and (3.20)

$$
\begin{aligned}
\bar{\Psi}\left(\zeta_{0}\right) & \leq \lim _{\rho \rightarrow 0}\left(C_{2} \rho^{\gamma}+\sup \left\{\mathcal{M} \Psi(\tilde{\zeta}) ; \tilde{\zeta} \in G_{\rho}\right\}\right) \\
& =\overline{\mathcal{M} \Psi}\left(\zeta_{0}\right) \leq \mathcal{M} \Psi\left(\zeta_{0}\right)<\Psi\left(\zeta_{0}\right)-\eta
\end{aligned}
$$

This contradiction proves the claim (3.25) and completes the proof that $\bar{\Psi}$ is a viscosity subsolution. 
b) Next we prove that $\underline{\Psi}$ is a viscosity supersolution. So we let $f$ be a $C^{2}$ function in a neighbourhood of $\mathcal{S}$ and we let $\zeta_{0} \in \mathcal{S}$ be such that $f \leq \underline{\Psi}$ on $\mathcal{S}$ and $f\left(\zeta_{0}\right)=\underline{\Psi}\left(\zeta_{0}\right)$. We want to show that

$$
F\left(D^{2} f\left(\zeta_{0}\right), D f\left(\zeta_{0}\right), f\left(\zeta_{0}\right), \underline{\Psi}, \zeta_{0}\right) \leq 0
$$

Since by Lemma 3.3 (ii) $\mathcal{M} \underline{\Psi}-\underline{\Psi} \leq 0$ everywhere, we see from (3.5) that this holds for $\zeta_{0} \in \ell_{1} \cup \ell_{2}$ and it suffices to show that

$$
\mathcal{L} f\left(\zeta_{0}\right) \leq 0 \quad \text { for } \zeta_{0} \in \mathcal{S}^{0} \cup[0, Q]
$$

and

$$
\mathcal{L}_{0} f\left(\zeta_{0}\right) \leq 0 \quad \text { for } \zeta_{0} \in[0, P]
$$

For $\varepsilon>0$ let $\widehat{w}=\widehat{w}_{\varepsilon, c}$ be an admissible control beginning with a constant consumption rate $c \geq 0$ and no transactions up to the first time $\tau=\tau_{\varepsilon}$ the process $Z^{c}(t)$ exits from

$$
K_{\varepsilon}=\left\{(s, x, y) ;\left|(s, x, y)-\left(0, x_{0}, y_{0}\right)\right|<\varepsilon\right\} \cap \widetilde{\mathcal{S}}
$$

where $\zeta_{0}=\left(x_{0}, y_{0}\right)$. Choose $\zeta_{n} \in K_{\varepsilon}$ such that $\zeta_{n} \rightarrow \zeta_{0}$ and $\Psi\left(\zeta_{n}\right) \rightarrow \underline{\Psi}\left(\zeta_{0}\right)$ as $n \rightarrow \infty$.

Then by combining Dynkin's formula with the dynamic programming principle ([Kr, Th. 6, p. 150]) we get for all $n$

$$
\begin{aligned}
& \Psi\left(\zeta_{n}\right) \geq E^{0, \zeta_{n}}\left[\int_{0}^{\tau} e^{-\delta t} \frac{c^{\gamma}}{\gamma} d t+e^{-\delta \tau} \Psi\left(X^{(\hat{w})}(\tau), Y^{(\hat{w})}(\tau)\right)\right] \\
& \geq E^{0, \zeta_{n}}\left[\int_{0}^{\tau} e^{-\delta t} \frac{c^{\gamma}}{\gamma} d t+e^{-\delta \tau} f\left(X^{(\hat{w})}(\tau), Y^{(\hat{w})}(\tau)\right)\right] \\
& =f\left(\zeta_{n}\right)+E^{0, \zeta_{n}}\left[\int_{0}^{\tau} e^{-\delta t}\left(L^{c} f\left(X^{(\hat{w})}(t), Y^{(\hat{w})}(t)\right)+\frac{c^{\gamma}}{\gamma}\right) d t\right] .
\end{aligned}
$$

We conclude that

$$
E^{0, \zeta_{n}}\left(\int_{0}^{\tau_{\varepsilon}} e^{-\delta t}\left\{L^{c} f\left(X^{(\hat{w})}(t), Y^{(\hat{w})}(t)\right)+\frac{c^{\gamma}}{\gamma}\right\} d t\right] \leq \Psi\left(\zeta_{n}\right)-f\left(\zeta_{n}\right) \quad \text { for all } n
$$

Taking the limit as $n \rightarrow \infty$ we obtain

$$
E^{0, \zeta_{0}}\left[\int_{0}^{\tau_{\varepsilon}} h(t) d t\right] \leq 0
$$

where

$$
h(t)=e^{-\delta t}\left(L^{c} f\left(X^{(\hat{w})}(t), Y^{(\hat{w})}(t)\right)+\frac{c^{\gamma}}{\gamma}\right) .
$$


By dividing the left hand side by $E^{0, \zeta_{0}}\left[\tau_{\varepsilon}\right]$ we get

$$
\begin{aligned}
& \frac{E^{0, \zeta_{0}}\left[\int_{0}^{\tau_{\varepsilon}} h(t) d t\right]}{E^{0, \zeta_{0}}\left[\tau_{\varepsilon}\right]}=\frac{E^{0, \zeta_{0}}\left[\int_{0}^{\tau_{\varepsilon}}(h(t)-h(0)) d t\right]+h(0) E^{0, \zeta_{0}}\left[\tau_{\varepsilon}\right]}{E^{0, \zeta_{0}}\left[\tau_{\varepsilon}\right]} \\
& \longrightarrow h(0) \quad \text { as } \varepsilon \rightarrow 0, \text { since } h(t) \text { is continuous at } t=0 .
\end{aligned}
$$

We conclude that

$$
h(0)=L^{c} f\left(\zeta_{0}\right)+\frac{c^{\gamma}}{\gamma} \leq 0
$$

for all $c \geq 0$ such that $\widehat{w}_{\varepsilon, c}$ is admissible for $\varepsilon$ small enough. If $\zeta_{0} \in \mathcal{S}^{0} \cup[0, Q]$ this is clearly the case for all $c \geq 0$ and therefore $(3.27)$ implies that $\mathcal{L} f\left(\zeta_{0}\right) \leq 0$. If $\zeta_{0} \in[0, P]$ the only such admissible $c$ is $c=0$. Therefore we get $\mathcal{L}_{0} f\left(\zeta_{0}\right) \leq 0$ in this case, as required.

Next we turn to the question of uniqueness. Our second main result in the section is the following:

\section{Theorem 3.8 (Comparison theorem)}

a) Suppose $u$ is a viscosity subsolution and $v$ is a viscosity supersolution of (3.4) and that $u$ and $v$ satisfy the estimates

$$
\begin{aligned}
& -C|x+y|^{\gamma} \leq u(x, y) \quad \text { for all } \quad(x, y) \in \mathcal{S} \\
& v(x, y) \leq C|x+y|^{\gamma} \quad \text { for all } \quad(x, y) \in \mathcal{S}
\end{aligned}
$$

for some constant $C<\infty$. Then

$$
u \leq v \quad \text { in } \mathcal{S}^{0}
$$

b) Moreover, if in addition

$$
v(x, y)=\liminf _{\substack{(\xi, \eta) \rightarrow(x, y) \\(\xi, \eta) \in \mathcal{S}^{0}}} v(\xi, \eta) \quad \text { for all }(x, y) \in \partial \mathcal{S}
$$

then

$$
u \leq v \quad \text { in } \mathcal{S}
$$

Corollary 3.9 Suppose $u$ and $v$ are two viscosity solutions of (3.4) satisfying (3.28) and (3.29). Then

$$
u=v \quad \text { in } \mathcal{S}^{0}
$$

and $u$ is continuous in $\mathcal{S}^{0}$. In particular, if (2.23) holds then the value function $\Psi$ is continuous on $\mathcal{S}^{0}$. 
Proof of Corollary 3.9. Since $u$ is a viscosity solution it follows that $\bar{u}$ is a viscosity subsolution and $\underline{u}$ is a viscosity supersolution and similarly for $v$. Hence, by Theorem 3.8,

$$
\bar{u} \leq \underline{v} \leq \bar{v} \leq \underline{u} \leq \bar{u} \quad \text { in } \mathcal{S}^{0} .
$$

This implies that

$$
\underline{u}=\bar{u}=\underline{v}=\bar{v} \quad \text { in } \quad \mathcal{S}^{0} .
$$

The last statement of Corollary 3.9 now follows from Theorem 3.7 and Corollary 2.2.

Proof of Theorem 3.8. The proof is based on the technique of Ishii (see [B], [CIL] and [IL]) and on the proofs of Lemma 3.12 of [AMS] and Theorem 5.7 in [AST]. Consequently we shall not give here a detailed proof but rather point out the special treatment required to handle the non local intervention operator $\mathcal{M}$.

Let $u$ and $v$ be as in Theorem 3.8. We first construct a strict supersolution of (3.4) by making a perturbation of $v$ :

Choose $\gamma^{\prime} \in(\gamma, 1)$ such that (see $\left.(1.23)\right)$

$$
\delta>\gamma^{\prime}\left[r+\frac{(\alpha-r)^{2}}{2 \sigma^{2}\left(1-\gamma^{\prime}\right)}\right]
$$

Put

$$
g(x, y)=(x+y)^{\gamma^{\prime}}
$$

and choose $\varepsilon>0$. Then

$$
\mathcal{M}(v+\varepsilon g) \leq \mathcal{M} v+\varepsilon \mathcal{M} g
$$

and hence

$$
\mathcal{M}(v+\varepsilon g)-(v+\varepsilon g) \leq(\mathcal{M} v-v)+\varepsilon(\mathcal{M} g-g) .
$$

Since $v$ is a supersolution we have

$$
\mathcal{M} v-v \leq 0
$$

Moreover, with $\zeta=(x, y)$,

$$
\begin{aligned}
(\mathcal{M} g-g)(\zeta) & =\sup _{\xi \neq 0}\{g(x-k-\xi-\lambda|\xi|, y+\xi)\}-g(x, y) \\
& =\sup _{\xi \neq 0}\left\{(x+y-k-\lambda|\xi|)^{\gamma^{\prime}}\right\}-(x+y)^{\gamma^{\prime}} \\
& \leq(x+y)^{\gamma^{\prime}}\left[\left(1-\frac{k}{x+y}\right)^{\gamma^{\prime}}-1\right] .
\end{aligned}
$$

Therefore, for each compact subset $C$ of $\mathcal{S} \backslash\{0\}$ there exists $\rho_{1}>0$ such that $(\mathcal{M} g-g)(\zeta) \leq-\rho_{1}$ for all $\zeta \in C$. So from (3.34) and (3.35) we get

$$
\mathcal{M}(v+\varepsilon g)-(v+\varepsilon g) \leq-\varepsilon \rho_{1} \quad \text { in } C .
$$


Now if we define the operator $L^{0}$ by (see (2.2))

$$
L^{0}=-\delta I+r x \frac{\partial}{\partial x}+\alpha y \frac{\partial}{\partial y}+\frac{1}{2} \sigma^{2} y^{2} \frac{\partial^{2}}{\partial y^{2}}
$$

where $I$ is the identity operator, then

$$
\begin{aligned}
& L^{0} g(x, y)=-\delta(x+y)^{\gamma^{\prime}}+(r x+\alpha y) \gamma^{\prime}(x+y)^{\gamma^{\prime}-1} \\
& \quad+\frac{1}{2} \sigma^{2} y^{2} \gamma^{\prime}\left(\gamma^{\prime}-1\right)(x+y)^{\gamma^{\prime}-2} \\
& =(x+y)^{\gamma^{\prime}}\left[-\delta+\gamma^{\prime} \frac{r x+\alpha y}{x+y}+\frac{1}{2} \sigma^{2} \gamma^{\prime}\left(\gamma^{\prime}-1\right) \frac{y^{2}}{(x+y)^{2}}\right] .
\end{aligned}
$$

If we put

$$
\eta=\frac{y}{x+y} \quad \text { so that } \quad \frac{x}{x+y}=1-\eta
$$

we get

$$
L^{0} g(x, y)=(x+y)^{\gamma^{\prime}}\left[-\delta+\gamma^{\prime} r+\gamma^{\prime}(\alpha-r) \eta+\frac{1}{2} \sigma^{2} \gamma^{\prime}\left(\gamma^{\prime}-1\right) \eta^{2}\right] .
$$

By (3.31) it follows that

$$
L^{0} g(x, y)<0 \quad \text { for all }(x, y) \neq(0,0)
$$

Consequently, on every compact $C$ of $\mathcal{S} \backslash\{0\}$ there exists $\rho_{2}>0$ such that

$$
L^{0} g(x, y)+\max _{c \geq 0}\left(-c \frac{\partial g}{\partial x}\right) \leq-\rho_{2} \quad \text { on } C .
$$

Therefore, since $v$ is a supersolution of (3.4) we conclude that on every compact $C$ of $\mathcal{S} \backslash\{0\}$ there exists $\rho>0$ such that

$$
v_{\varepsilon}:=v+\varepsilon g
$$

is a viscosity supersolution of

$$
F\left(D^{2} v_{\varepsilon}(\zeta), D v_{\varepsilon}(\zeta), v_{\varepsilon}(\zeta), v_{\varepsilon}, \zeta\right)=-\varepsilon \rho \quad \text { for } \zeta \in C
$$

Let us now prove the theorem by contradition. Assume that

$$
\sup _{\zeta \in \mathcal{S}}\{u(\zeta)-v(\zeta)\}>0 .
$$

Choose $\varepsilon>0$ such that

$$
\sup _{\zeta \in \mathcal{S}}\left\{u(\zeta)-v_{\varepsilon}(\zeta)\right\}>0
$$

Define

$$
h(\zeta):=u(\zeta)-v_{\varepsilon}(\zeta)
$$


Since $h$ is upper semicontinuous and tends to $-\infty$ when $|\zeta| \rightarrow \infty$, the set

$$
\operatorname{Argmax} h:=\{\bar{\zeta} ; h(\bar{\zeta})=\sup \{h(\zeta) ; \zeta \in \mathcal{S}\}\}
$$

is nonempty and compact in $\mathcal{S} \backslash\{0\}$. Choose an open set $G \subset \mathcal{S} \backslash\{0\}$ containing this compact ( $G$ open relative to $\mathcal{S}$ ) and with $\bar{G}$ compact. In order to get a contradition it suffices to prove that

$$
u \leq v_{\varepsilon} \quad \text { in } G .
$$

Thus we have reduced the problem to proving a comparison theorem for a strict supersolution $v_{\varepsilon}$ and a subsolution $u$ of (3.4) in an open subset $G$ of $\mathcal{S} \backslash\{0\}$ with compact closure $\bar{G}$, when the supremum of $u-v_{\varepsilon}$ is attained in $G$ only. This is proved by using Ishii's technique, adapted as in [B, Theorem 4.6] and in [AMS, Theorem 5.7] for the boundary conditions. We now explain this in more detail:

For $j=1,2, \ldots$ define, for $(\zeta, \eta) \in \mathcal{S} \times \mathcal{S}$,

$$
H_{j}(\zeta, \eta)=u(\zeta)-v_{\varepsilon}(\eta)-\frac{j}{2}|\zeta-\eta|^{2}
$$

and put

$$
m_{j}=\sup \left\{H_{j}(\zeta, \eta) ;(\zeta, \eta) \in \mathcal{S} \times \mathcal{S}\right\}
$$

$$
m=\sup \{h(\zeta) ; \zeta \in \mathcal{S}\} .
$$

Proceeding exactly as in $[\mathrm{AMS}]$ we obtain that there exist $\zeta_{j}, \eta_{j}$ in $\mathcal{S}$ such that

$$
m_{j}=H_{j}\left(\zeta_{j}, \eta_{j}\right)<\infty
$$

Moreover,

$$
j\left|\zeta_{j}-\eta_{j}\right|^{2} \rightarrow 0 \quad \text { as } j \rightarrow \infty
$$

and

$$
m_{j} \rightarrow m \quad \text { as } j \rightarrow \infty
$$

Suppose

Argmax $h$ is contained in $\mathcal{S}^{0}$

and choose $\hat{\zeta} \in \mathcal{S}^{0}$ such that

$$
m=h(\hat{\zeta})
$$

Then we get that

$$
\left(\zeta_{j}, \eta_{j}\right) \in \mathcal{S}^{0} \times \mathcal{S}^{0} \quad \text { for } j \text { large enough . }
$$

By applying [CIL, Theorem 3.2] we now obtain that there exist $2 \times 2$ matrices $P_{j}, Q_{j}$ such that

$$
\left(p_{j}, P_{j}\right) \in \bar{J}^{2,+} u\left(\zeta_{j}\right) \quad \text { and } \quad\left(q_{j}, Q_{j}\right) \in \bar{J}^{2,-} v_{\varepsilon}\left(\eta_{j}\right)
$$


and

$$
\left[\begin{array}{cc}
P_{j} & 0 \\
0 & -Q_{j}
\end{array}\right] \leq 3 j\left[\begin{array}{cc}
I & -I \\
-I & I
\end{array}\right]
$$

where

$$
p_{j}=j\left(\zeta_{j}-\eta_{j}\right) \quad \text { and } \quad q_{j}=p_{j} .
$$

Since $u$ is a subsolution and $v_{\varepsilon}$ is a strict supersolution we obtain

$$
\bar{F}\left(P_{j}, p_{j}, u, \zeta_{j}\right) \geq 0
$$

and

$$
\underline{F}\left(Q_{j}, q_{j}, v_{\varepsilon}, \eta_{j}\right) \leq-\varepsilon \rho .
$$

From (3.53) it follows that

$$
\Lambda\left(Q_{j}, q_{j}, v_{\varepsilon}, \eta_{j}\right)<0
$$

and proceeding as in [AMS] we obtain

$$
\Lambda\left(P_{j}, p_{j}, u, \zeta_{j}\right)-\Lambda\left(Q_{j}, q_{j}, v_{\varepsilon}, \eta_{j}\right)<0 .
$$

Consequently $\Lambda\left(P_{j}, p_{j}, u, \zeta_{j}\right)<0$ and from (3.52) we obtain that

$$
(\mathcal{M} u-u)\left(\zeta_{j}\right) \geq 0 \text {. }
$$

From (3.53) we have

$$
\left(\mathcal{M} v_{\varepsilon}-v_{\varepsilon}\right)\left(\eta_{j}\right) \leq-\varepsilon \rho<0 .
$$

Therefore, combining (3.55) and (3.56) we get

$$
m_{j}<u\left(\zeta_{j}\right)-v_{\varepsilon}\left(\eta_{j}\right)<\mathcal{M} u\left(\zeta_{j}\right)-\mathcal{M} v_{\varepsilon}\left(\eta_{j}\right)-\varepsilon \rho .
$$

Since $\zeta_{j}, \eta_{j} \rightarrow \hat{\zeta}$ and $u$ is usc, we obtain

$$
m<\liminf _{j \rightarrow \infty}\left[\mathcal{M} u\left(\zeta_{j}\right)-\mathcal{M} v_{\varepsilon}\left(\eta_{j}\right)\right] .
$$

Since $u$ is usc and $v_{\varepsilon}$ is lsc we see, after some reflections, that

$$
\limsup _{j \rightarrow \infty} \mathcal{M} u\left(\zeta_{j}\right) \leq \mathcal{M} u(\hat{\zeta})
$$

and

$$
\limsup _{j \rightarrow \infty}\left(-\mathcal{M} v_{\varepsilon}\left(\eta_{j}\right)\right) \leq-\mathcal{M} v_{\varepsilon}(\hat{\zeta}) .
$$

Hence we get the desired contradition

$$
\begin{aligned}
m & <\mathcal{M} u(\hat{\zeta})-\mathcal{M} v_{\varepsilon}(\hat{\zeta}) \\
& =\sup _{\xi_{1} \neq 0}\left\{u\left(\hat{x}^{\prime}\left(\xi_{1}\right), \hat{y}^{\prime}\left(\xi_{1}\right)\right)\right\}-\sup _{\xi_{2} \neq 0}\left\{v_{\varepsilon}\left(\hat{x}^{\prime}\left(\xi_{2}\right), \hat{y}^{\prime}\left(\xi_{2}\right)\right)\right\} \\
& \leq \sup _{\xi \neq 0}\left\{\left(u-v_{\varepsilon}\right)\left(\hat{x}^{\prime}(\xi), \hat{y}^{\prime}(\xi)\right)\right\} \\
& \leq \sup \left\{\left(u-v_{\varepsilon}\right)(\zeta) ; \zeta \in \mathcal{S}\right\}=m,
\end{aligned}
$$


where $\hat{\zeta}=(\hat{x}, \hat{y})$ and $($ see $(2.3))$

$$
\hat{x}^{\prime}(\xi)=\hat{x}-\xi-\lambda|\xi|-k, \quad \hat{y}^{\prime}(\xi)=\hat{y}+\xi .
$$

This contradiction shows that assumption (3.49) cannot hold. Therefore we must have

$$
\hat{\zeta} \in \operatorname{Argmax} h \cap \partial \mathcal{S} .
$$

To treat the boundary points we proceed exactly as in the proof of Theorem 5.7 of [AST], which itself is based on the proof of Theorem 4.6 in [B] (see Appendix, p. 166).

From (3.30) there exists a sequence $\left\{\zeta_{j}\right\} \subset \mathcal{S}^{0} \cap G$ converging to $\hat{\zeta}$ such that $v_{\varepsilon}\left(\zeta_{j}\right) \rightarrow$ $v_{\varepsilon}(\hat{\zeta})$ when $j \rightarrow \infty$. Let $\varepsilon_{j}=\left|\zeta_{j}-\hat{\zeta}\right|$. The idea is now to introduce the test function

$$
w_{j}(\zeta, \eta)=u(\zeta)-v_{\varepsilon}(\eta)-\theta_{j}(\zeta, \eta) ; \quad(\zeta, \eta) \in \mathcal{S} \times \mathcal{S}
$$

where

$$
\theta_{j}(\zeta, \eta)=\frac{|\zeta-\eta|^{2}}{2 \varepsilon_{j}}+\frac{1}{4}\left(\frac{d(\eta)-d(\zeta)}{d\left(\zeta_{j}\right)}-1\right)^{4}+\frac{1}{4}|\zeta-\hat{\zeta}|^{4}
$$

Here $d(\eta)$ denotes the distance from $\eta$ to $\partial \mathcal{S}$ and similarly for $d(\zeta), d\left(\zeta_{j}\right)$.

Following exactly the same steps as in $[\mathrm{AST}]$ and treating the term $\mathcal{M} u-u$ as we did before, we obtain a contradiction also in the case (3.61). This shows that (3.40) cannot hold and this completes the proof of Theorem 3.8 .

We summarize the results of this section in the following:

Theorem 3.10 Let $\Psi(x, y)$ be the value function given by (1.17). Suppose (2.23) holds, i.e.

$$
\delta>\gamma \alpha .
$$

Then $\Psi$ is continuous on $\mathcal{S}^{0}$ and $\Psi$ is the unique viscosity solution of (3.4) with the property that there exists $C<\infty$ such that

$$
|\Psi(x, y)| \leq C|x+y|^{\gamma} \quad \text { for all }(x, y) \in \mathcal{S}
$$

\section{Numerical results}

In this section we present the result of a numerical method used to approximate the viscosity solution of (3.4) in the case when the solvency region is $\mathcal{S}_{+}=[0, \infty) \times[0, \infty)$ (see Remark 1.1). This method will be detailed in a forthcoming paper [CØS]. It is based on an iterative method which permits us to obtain the QVHJBI as a limit of variational HJB inequalities. Each variational inequality is approximated by a finite difference scheme and then solved by a Howard algorithm. 


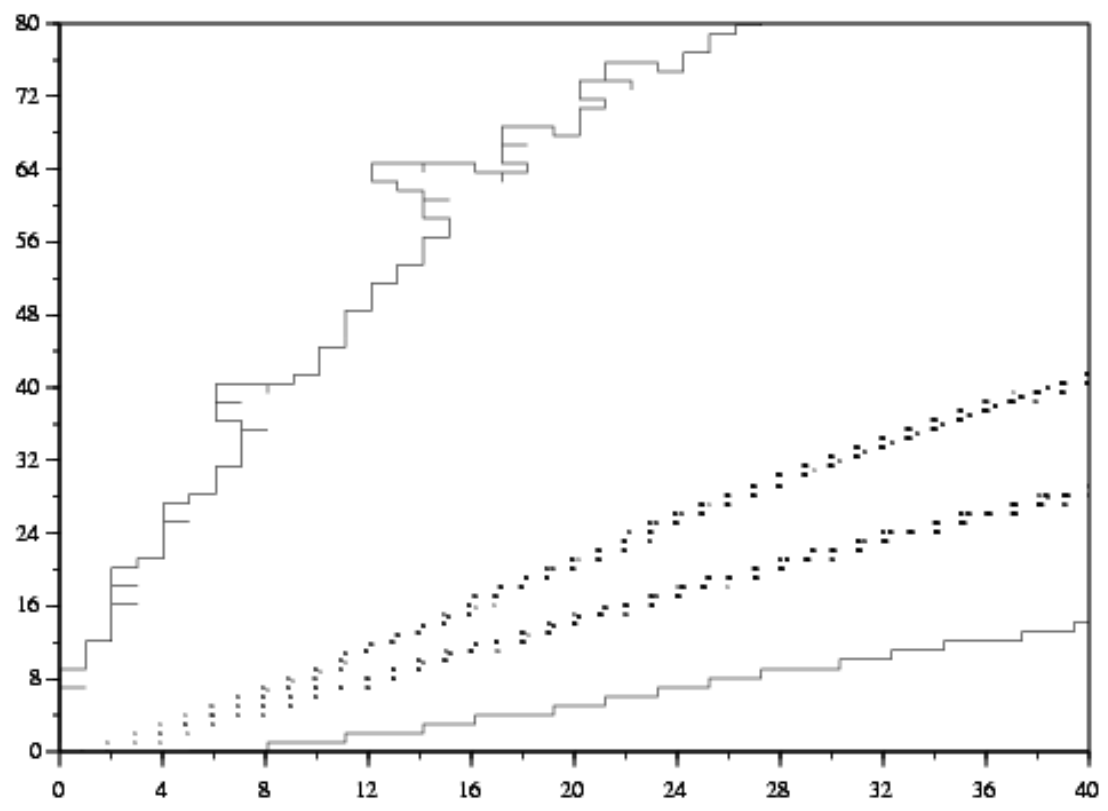

Figure 4: Optimal policy for $k=0.1, \lambda=0.01, \sigma=0.3, r=0.07, \delta=0.1, \gamma=0.3, \alpha=0.11$

Figure 4 represents the boundary of the no-transaction region for the following values of the parameters: $k=0.1, \lambda=0.01, \sigma=0.3, r=0.07, \delta=0.1, \gamma=0.3, \alpha=0.11$. The dotted lines (situated inside the no-transaction region) represent the set of positions reached after a transaction.

It is natural to ask if it is possible to obtain more information about the shape of the no-transaction region NT, both for this choice $\mathcal{S}_{+}$of solvency region and for the choice $\mathcal{S}$ given by (1.9). As mentioned in the introduction we know that if $k=0$, then NT is bounded by two straight lines from the origin [DN] (see Figure 2). If $k>0$, is NT still bounded by two curves? If so, what can be said about the form of these curves? Can they be given an explicit description?

Remark For results on viscosity solutions of QVIs corresponding to impulse control problems (which, however, do not apply to our situation), see $[\mathrm{I}],[\mathrm{P}]$ and $[\mathrm{TY}]$.

\section{Acknowledgements}

We wish to thank Marianne Akian, Guy Barles, Nils Christian Framstad and Kristin Reikvam for very helpful comments and fruitful discussions. This work was partially supported by the French-Norwegian cooperation project Aur 99-050. 


\section{References}

[AMS] M. Akian, J.L. Menaldi and A. Sulem: On an investment-consumption model with transaction costs. SIAM J. Control \& Opt. 34 (1996), 329-364.

[AST] M. Akian, A. Sulem and M.I. Taksar: Dynamic optimization of long term growth rate for a portfolio with transaction costs and logarithmic utility. Math. Finance 11 (2001), 153-188.

[B] G. Barles: Solutions de viscosité des équations de Hamilton-Jacobi. Math. \& Appl. 17. Springer-Verlag 1994.

[BL] A. Bensoussan and J.-L. Lions: Impulse Control and Quasi-Variational Inequalities. Gauthier-Villars 1984.

[BØ] K.A. Brekke and B. Øksendal: A verification theorem for combined stochastic control and impulse control. In Decreusefond et al (eds.): Stochastic Analysis and Related Topics, Vol. 6. Birkhäuser 1997, pp. 211-220.

[BP] T.R. Bielecki and S.R. Pliska: Risk sensitive asset management with transaction costs. Finance 8 Stochast. 4 (2000), 1-33.

[CIL] M.G. Crandall, H. Ishii and P.-L. Lions: User's guide to viscosity solutions of second order partial differential equations. Bulletin Amer. Math. Soc. 27 (1992), 1-67.

[CZ1] A. Cadenillas and F. Zapatero: Optimal central bank intervention in the foreign exchange market. J. Economic Theory 87 (1999), 218-242.

[CZ2] A. Cadenillas and F. Zapatero: Classical and impulse stochatic control of the exchange rate using interest rates and reserves. Math. Finance 10 (2000), 141-156.

[CØS] J.-P. Chancelier, B. Øksendal and A. Sulem: Combined stochastic control and optimal stopping, with application to portfolio optimization under fixed transaction costs. Preprint, Dept. of Mathematics, University of Oslo, 16, 2000.

[DN] M.H.A. Davis and A. Norman: Portfolio selection with transaction costs. Math. Oper. Res 15 (1990), 676-713.

[EH] J.E. Eastham and K.J. Hastings: Optimal impulse control of portfolios. Math. Oper. Res. 13 (1988), 588-605.

[FØS1] N.C. Framstad, B. Øksendal and A. Sulen: Optimal consumption and portfolio in a jump diffusion market. In A. Shiryaev and A. Sulem (editors): Workshop on Mathematical Finance. INRIA, Paris 1998. 
[FØS2] N.C. Framstad, B. Øksendal and A. Sulen: Optimal consumption and portfolio in a jump diffusion market with proportional transaction costs. J. Mathematical Economics (to appear).

[I] K. Ishii: Viscosity solutions of nonlinear second order elliptic PDEs associated with impulse control problems. Funkcial. Ekvac. 36 (1993), 123-141.

[IL] H. Ishii and P.L. Lions: Viscosity solutions of fully nonlinear second-order elliptic partial differential equations. Journal of Diff. Eq. 83 (1990), 26-78.

[IW] N. Ikeda and S. Watanabe: Stochastic Differential Equations and Diffusion Processes. Second Edition. North-Holland/Kodansha 1989.

[K] R. Korn: Portfolio optimization with strictly positive transaction costs and impulse control. Finance \& Stochast. 2 (1998), 85-114.

[Kr] N.V. Krylov: Controlled Diffusion Processes. Springer-Verlag 1980.

[M] R.C. Merton: Optimum consumption and portfolio rules in a continuous time model. J. Economic Theory 3 (1971), 373-413.

[MØ] G. Mundaca and B. Øksendal: Optimal stochastic intervention control with application to the exchange rate. J. Math. Economics 29 (1998), 225-243.

[Ø] B. Øksendal: Stochastic Differential Equations. 5th edition. Springer-Verlag 2000.

[ØS] B. Øksendal and A. Sulem: Introduction to Impulse Control Theory and Applications to Economics. Lecture Notes, INRIA/University of Oslo 2000.

[P] B. Perthame: Recent results on the quasi-variational inequality of the impulse control. Contributions to Nonlinear Partial Differential Equations, Vol. II, Pitman Res. Notes Math. Ser., 155 (Paris, 1985), 210-219.

[RØ] K. Reikvam and B. Øksendal: Viscosity solutions of optimal stopping problems. Stochastics and Stochastics Reports 62 (1998), 285-301.

[SS] S.E. Shreve and H.M. Soner: Optimal investment and consumption with transaction costs. Ann. Appl. Probab. 4 (1994), 609-692.

[TY] S. Tang and J. Yong: Finite horizon stochastic optimal switching and impulse controls with a viscosity solution approach. Stochastics and Stochastics Reports 45 (1993), 145-176. 